\title{
SPATIAL RANDOM PERMUTATIONS AND INFINITE CYCLES
}

\author{
VOLKER BETZ AND DANIEL UELTSCHI
}

\begin{abstract}
We consider systems of spatial random permutations, where permutations are weighed according to the point locations. Infinite cycles are present at high densities. The critical density is given by an exact expression. We discuss the relation between the model of spatial permutations and the ideal and interacting quantum Bose gas.

Keywords: Spatial random permutations, infinite cycles, Bose-Einstein condensation.

2000 Math. Subj. Class.: 60K35, 82B20, 82B26, 82B41.
\end{abstract}

\section{Contents}

1. Introduction

2. The model in finite volume

1

3. The model in infinite volume

3.2. An extension theorem

3.3. Permutation cycles and probability measure 9

3.4. Finite vs infinite volume 10

4. A regime without infinite cycles 10

5. The one-body model 11

5.1. Occurrence of infinite cycles

5.2. Fourier representation for spatial permutations 13

6. The quantum Bose gas 17

6.1. Feynman-Kac representation of the Bose gas

6.2. Discussion: Relevant interactions for spatial permutations 19

7. A simple model of spatial random permutations with interactions

7.1. Approximation and definition of the model

7.2. Pressure and critical density 21

7.3. Occurrence of infinite cycles

Appendix A. Macroscopic occupation of the zero Fourier mode.

Appendix B. Convexity and Fourier positivity 29

References

\section{INTRODUCTION}

This article is devoted to random permutations on countable sets that possess a spatial structure. Let $\boldsymbol{x}$ be a finite set of points $x_{1}, \ldots, x_{N} \in \mathbb{R}^{d}$, and let $\mathcal{S}_{N}$ be the set of permutations of $N$ elements. We are interested in probability measures on $\mathcal{S}_{N}$ where permutations with long jumps are discouraged. The main example deals with "Gaussian" weights, where the probability of $\pi \in \mathcal{S}_{N}$ is proportional to

$$
\exp \left\{-\frac{1}{4 \beta} \sum_{i=1}^{N}\left|x_{i}-x_{\pi(i)}\right|^{2}\right\},
$$


with $\beta$ a parameter. But we are also interested in more general weights on permutations. In addition, we want to allow the distribution of points to be random and to depend on the permutations.

We are mostly interested in the existence and properties of such measures in the thermodynamic limit. That is, assuming that the $N$ points $\boldsymbol{x}$ belong to a cubic box $\Lambda \subset \mathbb{R}^{d}$, we consider the limit $|\Lambda|, N \rightarrow \infty$, keeping the density $\rho=N /|\Lambda|$ fixed. The main question is the possible occurrence of infinite cycles. As will be seen, infinite cycles occur when the density is larger than a critical value.

Mathematicians and physicists have devoted many efforts to investigating properties of non-spatial random permutations, when all permutations carry equal weight. In particular, a special emphasis has been put on the study of longest increasing subsequences [1, 3] and their implications for such diverse areas as random matrices [3, 21], GromovWitten theory [22] or polynuclear growth [8], and spectacular results have been obtained. The situation is very different for random permutations involving spatial structure; we are only aware of the works [16, 17, 10] (and [11]). This lack of attention is odd since spatial random permutations are natural and appealing notions in probability theory; it becomes even more astonishing when considering that they play an important rôle in the study of quantum bosonic systems: Feynman [9] and Penrose and Onsager [23] pointed out the importance of long cycles for Bose-Einstein condensation, and later Sütő clarified the notion of infinite cycles, also showing that infinite, macroscopic cycles are present in the ideal Bose gas [25, 26]. These works, however, never leave the context of quantum mechanics. We believe that the time is ripe for introducing a general mathematical framework of spatial random permutations. The goal of this article is to clarify the setting and the open questions, and also to present some results.

In Section 2, we introduce a model for spatial random permutations in a bounded domain $\Lambda \subset \mathbb{R}^{d}$. As stated above, the intuition is to suppress permutations with large jumps. We achieve this by assigning a "one-body energy" of the form $\sum_{i} \xi\left(x_{i}-x_{\pi(i)}\right)$ to a given permutation $\pi$ on a finite set $\boldsymbol{x}$. The one-body potential $\xi$ is nonnegative, spherically symmetric, and typically monotonically increasing, although we will allow more general cases. In addition, we will introduce "many-body potentials" depending on several jumps, as well as a weight for the points $\boldsymbol{x}$.

As usual, the most interesting mathematical structures will emerge in the thermodynamic limit $|\Lambda|, N \rightarrow \infty$, where $N$ is the number of points in $\boldsymbol{x}$. The ambitious approach is to consider and study the infinite volume limit of probability measures. The limiting measure should be a well-defined joint probability measure on countably infinite (but locally finite) sets $\boldsymbol{x} \subset \mathbb{R}^{d}$, and on permutations of $\boldsymbol{x}$. To establish such a limit seems fairly difficult; as an alternative one can settle for constructing an infinite volume measure for permutations only, with a fixed $\boldsymbol{x}$ chosen according to some point process. We provide a framework for doing so in Section 3, and give a natural criterion for the existence of the infinite volume limit (it is a generalisation of the one given in [11]). This criterion is trivially fulfilled if the interaction prohibits jumps greater than a certain finite distance; however, its verification for the physically most interesting cases remains an open problem.

Another option for taking the thermodynamic limit is to focus on the existence of the limiting distribution of one special random variable as $|\Lambda|, N \rightarrow \infty$. Motivated by its relevance to Bose-Einstein condensation, our choice of random variable is the probability of the existence of long cycles; more precisely, we will study the fraction of indices that lie in a cycle of macroscopic length. The general intuition is that in situations where points are sparse (low density), or where moderately long jumps are strongly discouraged 
(high temperature), the typical permutation is a small perturbation of the identity map, and there are no infinite cycles. In Section 4 we give a criterion (that corresponds to low densities, resp. high temperatures) for the absence of infinite cycles.

On the other hand, infinite cycles are usually present for high density. The density where existence of infinite cycles first occurs is called the critical density. We establish the occurrence of infinite, macroscopic cycles in Section 5 for the case where only the one-body potential is present, and where we average over the point configurations $\boldsymbol{x}$ in a suitable way. An especially pleasing aspect of the result is the existence of a simple, exact formula for the critical density. It turns out to be nothing else than the critical density of the ideal Bose gas, first computed by Einstein in 1925! The experienced physicist may shrug this fact off in hindsight. However, it is a priori not apparent why quantum mechanics should be useful in understanding this problem, and it is fortunate that much progress has been achieved on bosonic systems over the years. Of direct relevance here is Sütö's study of the ideal gas [26], and the work of Buffet and Pulé on distributions of occupation numbers [6].

Section 6 is devoted to the relation between models of spatial random permutations and the Feynman-Kac representation of the Bose gas. We are particularly interested in the effect of interactions on the Bose-Einstein condensation. While this question has been largely left to numericians, experts in path-integral Monte-Carlo methods, we expect that weakly interacting bosons can be exactly described by a model of spatial permutations with two-body interactions. An interesting open problem is to establish this fact rigorously. Numerical simulations of the model of spatial permutations should be rather easy to perform, and they should help us to understand the phase transition to a Bose condensate.

In Section 7 we simplify the interacting model of Section 6. It turns out that the largest terms contributing to the interactions between permutation jumps are due to cycles of length 2. Retaining this contribution only, we obtain a toy model of interacting random permutations that is simple enough to handle, but which allows to explore some of the effects of interactions on Bose-Einstein condensation. In particular, we are able to compute the critical temperature exactly. It turns out to be higher than the non-interacting one and to deviate linearly in the scattering length of the interaction potential. This is in qualitative agreement with the findings of the physical community [2, 14, 15, 20]. In addition, we show that infinite cycles occur in our toy model whenever the density is sufficiently high. However, our condition on the density is not optimal - higher than the critical density of our interacting model. Our condition is not optimal. We expect the existence of infinite cycles right down to the interacting critical density, but this is yet another open problem.

Acknowledgments. We are grateful to many colleagues for discussions over a rather long period of time. In particular, we would like to mention Stefan Adams, Michael Aizenman, Marek Biskup, Jürg Fröhlich, Daniel Gandolfo, Gian Michele Graf, Martin Hairer, Roman Kotecký, Alain Joye, Elliott Lieb, Bruno Nachtergaele, Charles-Édouard Pfister, Suren Poghosyan, Jose Luis Rodrigo, Jean Ruiz, Benjamin Schlein, Robert Seiringer, Herbert Spohn, Andras Sütő, Florian Theil, Jochen Voss, Valentin Zagrebnov, and Hans Zessin. V.B. is supported by the EPSRC fellowship EP/D07181X/1. D.U. is supported in part by the grant DMS-0601075 of the US National Science Foundation. 


\section{The MOdel In Finite VOlume}

Let $\Lambda$ be a bounded open domain in $\mathbb{R}^{d}$, and let $V$ denote its volume (Lebesgue measure). The state space of our model is the cartesian product

$$
\Omega_{\Lambda, N}=\Lambda^{N} \times \mathcal{S}_{N}
$$

where $\mathcal{S}_{N}$ is the symmetric group of permutations of $\{1, \ldots, N\}$. The state space $\Omega_{\Lambda, N}$ can be equipped with the product $\sigma$-algebra of the Borel $\sigma$-algebra for $\Lambda^{N}$, and the discrete $\sigma$-algebra for $\mathcal{S}_{N}$. An element $\left(x_{1}, \ldots, x_{N}\right) \times \pi \in \Omega_{\Lambda, N}$ is viewed as a spatial random permutation in the sense that $x_{j}$ is mapped to $x_{\pi(j)}$ for all $j$. Figure 1 illustrates this. The probability measure on $\Omega_{\Lambda, N}$ is obtained in the usual way of statistical mechanics:

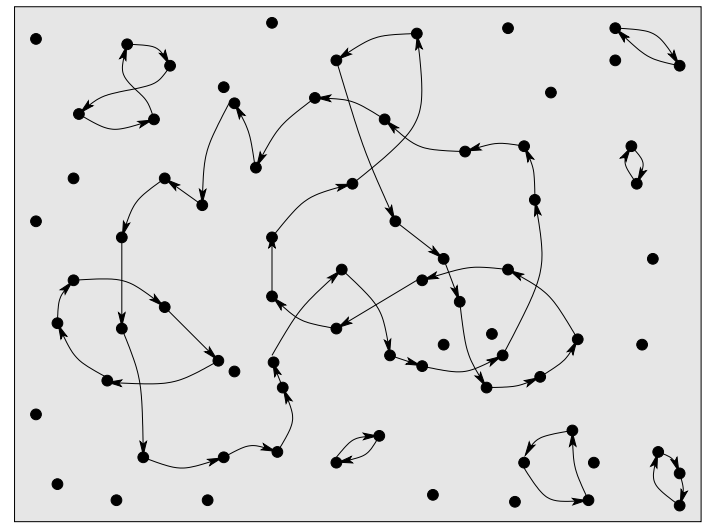

FigURE 1. Illustration for a random set of points $\boldsymbol{x}$, and for a permutation $\pi$ on $\boldsymbol{x}$. Isolated points are sent onto themselves.

a reference measure, in our case the product of Lebesgue measure on $\Lambda^{N}$ and uniform measure on permutations, is perturbed by a density given by the exponential of a Hamiltonian, i.e. a function $H: \Omega_{\Lambda, N} \rightarrow(-\infty, \infty]$. We will shortly specify the shape of relevant Hamiltonians.

We are interested in properties of permutations rather than positions, and we only consider random variables on $\mathcal{S}_{N}$. We consider two different expectations: $E_{\boldsymbol{x}}$, when positions $\boldsymbol{x} \in \Lambda^{N}$ are fixed; and $E_{\Lambda, N}$, when we average over positions. For this purpose we introduce the partition functions

$$
\begin{aligned}
& Y(\boldsymbol{x})=\sum_{\pi \in \mathcal{S}_{N}} \mathrm{e}^{-H(\boldsymbol{x}, \pi)} \\
& Z(\Lambda, N)=\frac{1}{N !} \int_{\Lambda^{N}} Y(\boldsymbol{x}) \mathrm{d} \boldsymbol{x} .
\end{aligned}
$$

In the last line, $\mathrm{d} \boldsymbol{x}$ denotes the Lebesgue measure on $\mathbb{R}^{d N}$. The factor $1 / N$ ! implies that $Z(\Lambda, N) \sim \mathrm{e}^{V q}$ for large $V, N$, and for "reasonable" Hamiltonians - a desirable property in statistical mechanics. Then, for $\theta: \mathcal{S}_{N} \rightarrow \mathbb{R}$ a random variable on the set of permutations, we define

$$
E_{\boldsymbol{x}}(\theta)=\frac{1}{Y(\boldsymbol{x})} \sum_{\pi \in \mathcal{S}_{N}} \theta(\pi) \mathrm{e}^{-H(\boldsymbol{x}, \pi)}
$$


and

$$
\begin{aligned}
E_{\Lambda, N}(\theta) & =\frac{1}{Z(\Lambda, N) N !} \int_{\Lambda^{N}} \mathrm{~d} \boldsymbol{x} \sum_{\pi \in \mathcal{S}_{N}} \theta(\pi) \mathrm{e}^{-H(\boldsymbol{x}, \pi)} \\
& =\frac{1}{Z(\Lambda, N) N !} \int_{\Lambda^{N}} E_{\boldsymbol{x}}(\theta) Y(\boldsymbol{x}) \mathrm{d} \boldsymbol{x} .
\end{aligned}
$$

We will be mostly interested in the possible occurrence of long cycles. Thus we introduce a random variable that measures the density of points in cycles of length between $m$ and $n$ :

$$
\varrho_{m, n}(\pi)=\frac{1}{V} \#\left\{i=1, \ldots, N: m \leqslant \ell_{i}(\pi) \leqslant n\right\} .
$$

Here, $\ell_{i}(\pi)$ denotes the length of the cycle that contains $i$; that is, $\ell_{i}(\pi)$ is the smallest number $n \geqslant 1$ such that $\pi^{(n)}(i)=i$. We also have

$$
\varrho_{m, n}(\pi)=\frac{1}{V} \sum_{i=1}^{N} \chi_{[m, n]}\left(\ell_{i}(\pi)\right)
$$

with $\chi_{I}$ denoting the characteristic function for the interval $I$.

We denote by $\mathcal{R}_{N}$ the space of random variables on $\mathcal{S}_{N}$ that are invariant under transpositions. That is, $\theta \in \mathcal{R}_{N}$ satisfies

$$
\theta\left(\sigma^{-1} \pi \sigma\right)=\theta(\pi)
$$

for all $\sigma, \pi \in \mathcal{S}_{N}$. Random variables in $\mathcal{R}_{N}$ have the useful property that they do not depend on the way the set $\boldsymbol{x}=\left\{x_{1}, \ldots, x_{N}\right\}$ is labeled. Instead, they only depend on the set $\boldsymbol{x}$ itself, and in that sense are the most natural quantities to study. Notice that $\varrho_{m, n} \in \mathcal{R}_{N}$.

We now discuss the form of relevant Hamiltonians. $H$ is given by the sum

$$
H(\boldsymbol{x}, \pi)=H^{(1)}(\boldsymbol{x}, \pi)+\sum_{k \geqslant 2} H^{(k)}(\boldsymbol{x}, \pi)+G(\boldsymbol{x}),
$$

where the terms satisfy the following properties. Let $\boldsymbol{x}=\left(x_{1}, \ldots, x_{N}\right)$.

- The one-body Hamiltonian $H^{(1)}$ has the form

$$
H^{(1)}(\boldsymbol{x}, \pi)=\sum_{i=1}^{N} \xi\left(x_{i}-x_{\pi(i)}\right) .
$$

We suppose that $\xi$ is a spherically symmetric function $\mathbb{R}^{d} \rightarrow[0, \infty]$, that $\xi(0)=0$, and that $\mathrm{e}^{-\xi}$ is integrable.

- The $k$-body term $H^{(k)}: \Omega_{\Lambda, N} \rightarrow \mathbb{R}$ can be negative; is has the form

$$
H^{(k)}(\boldsymbol{x}, \pi)=\sum_{\substack{A \subset\{1, \ldots, N\} \\|A|=k}} V\left(\left(x_{j}, x_{\pi(j)}\right)_{j \in A}\right) .
$$

- The function $G: \mathbb{R}^{d N} \rightarrow \mathbb{R}$ depends on the points only. It has no effect on the expectation $E_{\boldsymbol{x}}$, but it modifies the expectation $E_{\Lambda, N}$.

We will discuss in Section 6 the links between spatial random permutations and the quantum Bose gas. We will see that the physically relevant terms are $\xi(x)=|x|^{2} / 4 \beta$, that the interactions are two-body $\left(H^{(k)}=0\right.$ for $\left.k>2\right)$, and that $G(\boldsymbol{x}) \equiv 0$. From the mathematical point of view it is interesting to consider a more general setting. In particular, we can restrict the jumps by setting $\xi(x)=\infty$ for $|x|$ bigger than some cutoff 
distance $R$. The effect of $G$ is to modify the typical sets of points. We can choose it such that $Y(\boldsymbol{x}) \equiv 1$. We refer to this case as "Poisson", since positions are independent of each other, and they are uniformly spread. The point process for the Bose gas is not Poisson, however. The fluctuations of the number of points in a subdomain were studied in [18]. They were shown to satisfy a large deviation principle with a rate function that is different than Poisson's.

\section{The MOdel IN INFinite VOLUME}

As usual, the most interesting structures emerge in the infinite volume limit $V \rightarrow \infty$ or, more precisely, the thermodynamic limit $V \rightarrow \infty, N=\rho V$. The easiest way to take this limit is to consider a fixed random variable, e.g. $\varrho_{a, b}(\pi)$ from $(2.5)$, and study its distribution as $V \rightarrow \infty$ and $N=\rho V$. We will indeed do this in Section 5 an advantage of this approach is that we do not have to worry about infinite volume probability measures. But these infinite volume measures are very interesting objects to study directly, in the same spirit as when constructing infinite volume Gibbs measures. We advocate this point of view in the present section, and introduce a framework for spatial permutations in unbounded domains.

3.1. The $\sigma$-algebra. The present and the following subsection contain preparatory results about permutations on $\mathbb{N}$. We introduce the "cylinder sets" $B_{i, j}$ that consist of all permutations where $i \in \mathbb{N}$ is sent to $j \in \mathbb{N}$ :

$$
B_{i, j}=\left\{\pi \in \mathcal{S}_{\mathbb{N}}: \pi(i)=j\right\} .
$$

Let $\Sigma^{\prime}$ denote the collection of finite intersections of cylinder sets and their complements. One can check that it is closed under finite intersections, and also that the difference of two sets is equal to a finite union of disjoint sets. Such a family of sets is called a semiring by probabilists. Semirings are useful because they are easy to build from basic sets, and because premeasures on semirings can be extended to measures by the CarathéodoryFréchet theorem. Let $\Sigma$ be the $\sigma$-algebra generated by the $B_{i, j}$.

We start by proving a structural lemma that we shall use when extending finite volume measures to an infinite volume one. For $A_{1}, A_{1}^{\prime}, \ldots, A_{m}, A_{m}^{\prime} \subset \mathbb{N}$, let us define

$$
B_{A_{1}^{\prime} \ldots A_{m}^{\prime}}^{A_{1} \ldots A_{m}}=\left\{\pi \in \mathcal{S}_{\mathbb{N}}: \pi^{-1}(i) \in A_{i} \text { and } \pi(i) \in A_{i}^{\prime}, 1 \leqslant i \leqslant m\right\} .
$$

One easily checks that any element of the semiring can be represented by a set of the form (3.2). Also, the intersection of two such sets satisfies

$$
B_{A_{1}^{\prime} \ldots A_{m}^{\prime}}^{A_{1} \ldots A_{m}} \cap B_{C_{1}^{\prime} \ldots C_{m}^{\prime}}^{C_{1} \ldots C_{m}}=B_{A_{1}^{\prime} \cap C_{1}^{\prime} \ldots A_{m}^{\prime} \cap C_{m}^{\prime}}^{A_{1} \cap C_{1} \ldots A_{m} \cap C_{m}}
$$

Lemma 3.1. Let $A_{1}, A_{1}^{\prime}, A_{2}, A_{2}^{\prime}, \ldots$ be finite subsets of $\mathbb{N}$. If

$$
B_{A_{1}^{\prime} \ldots A_{m}^{\prime}}^{A_{1} \ldots A_{m}} \neq \emptyset
$$

for all finite $m$, then $\lim _{m \rightarrow \infty} B_{A_{1}^{\prime} \ldots A_{m}^{\prime}}^{A_{1} \ldots A_{m}} \neq \emptyset$.

It is crucial that both $A_{i}$ and $A_{i}^{\prime}$ be finite for all $i$; counter-examples are easily found otherwise. For instance, choose $A_{1}=\mathbb{N}, A_{i}=\{i-1\}$ for $i>1$, and $A_{i}^{\prime}=\{i+1\}$ for all $i$; then each finite intersection is non-empty. The infinite intersection is empty, on the other hand, since there is no possibility left for the preimage of 1 . Similarly, choosing $A_{1}^{\prime}=\mathbb{N}$, $A_{i}^{\prime}=\{i-1\}$ for $i>1$ and $A_{i}=\{i+1\}$ does not leave a possible image for 1 . These two cases should be kept in mind when reading the proof. 
A claim similar to Lemma 3.1 and Theorem 3.2 was proposed in [1]. The proof there contains a little flaw that is corrected here.

Proof. We write $B_{a^{\prime}}^{a}$ instead of $B_{\left\{a^{\prime}\right\}}^{\{a\}}$, etc... We have

$$
B_{A_{1}^{\prime} \ldots A_{m}^{\prime}}^{A_{1} \ldots A_{m}}=\bigcup_{\substack{a_{1}, \ldots, a_{m} \\ a_{1}^{\prime}, \ldots, a_{m}^{\prime}}} B_{a_{1}^{\prime} \ldots a_{m}^{\prime}}^{a_{1} \ldots a_{m}} .
$$

The union is over $a_{1} \in A_{1}, \ldots, a_{m}^{\prime} \in A_{m}^{\prime}$, with the restriction that $a_{i} \neq a_{j}$ and $a_{i}^{\prime} \neq a_{j}^{\prime}$ for $i \neq j$. The union is disjoint, $B_{a_{1}^{\prime} \ldots a_{m}^{\prime}}^{a_{1} \ldots a_{m}} \neq \emptyset$, and

$$
B_{a_{1}^{\prime} \ldots a_{m+1}^{\prime}}^{a_{1} \ldots a_{m+1}} \subset B_{a_{1}^{\prime} \ldots a_{m}^{\prime}}^{a_{1} \ldots a_{m}}
$$

Permutations are charaterised by a "limiting set", namely

$$
\{\pi\}=B_{a_{1}^{\prime} a_{2}^{\prime} \cdots}^{a_{1} a_{2} \ldots}
$$

where $\left\{a_{i}\right\},\left\{a_{i}^{\prime}\right\}$ are given by

$$
\pi^{-1}(i)=a_{i} \quad \text { and } \quad \pi(i)=a_{i}^{\prime}
$$

Conversely, given $\left\{a_{i}\right\},\left\{a_{i}^{\prime}\right\}, B_{a_{1}^{\prime} a_{2}^{\prime} \ldots}^{a_{1} a_{2} \ldots}$ is either empty, or it contains the permutation $\pi$ that satisfies (3.7). We now check that the decomposition (3.4) yields at least one non-empty limiting set.

The sets $B_{a_{1}^{\prime} \ldots a_{m}^{\prime}}^{a_{1} \ldots a_{m}}$ that appear in 3.4 can be organised as a tree. The root is $\mathcal{S}_{\mathbb{N}}$. The sets with $m=1$ are connected to $\mathcal{S}_{\mathbb{N}}$, i.e. all the sets $B_{a_{1}^{\prime}}^{a_{1}}, a_{1} \in A_{1}$ and $a_{1}^{\prime} \in A_{1}^{\prime}$. The sets with $m=2$ are connected to those with $m=1$. Precisely, the sets $B_{a_{1}^{\prime} a_{2}^{\prime}}^{a_{1} a_{2}}, a_{2} \in A_{2}$ and $a_{2}^{\prime} \in A_{2}^{\prime}$, are connected to $B_{a_{1}^{\prime}}^{a_{1}}$. And so on... There are infinitely many vertices, but finitely many vertices at finite distance from the root.

We can select a limiting set as follows. Let $\ell_{a_{1}^{\prime} \ldots a_{m}^{\prime}}^{a_{1} \ldots a_{m}}$ denote the length of the longest path descending from $B_{a_{1}^{\prime} \ldots a_{m}^{\prime}}^{a_{1} \ldots a_{m}}$. For each $m$, there is at least one $\left\{a_{i}\right\},\left\{a_{i}^{\prime}\right\}$ such that $\ell_{a_{1}^{\prime} \ldots a_{m}^{\prime}}^{a_{1} \ldots a_{m}}=\infty$. (Otherwise the tree cannot have infinitely many vertices, since the sets $A_{1}, A_{1}^{\prime}, \ldots$ have finite cardinality.) Further, there exists $a_{m+1} \in A_{m+1}$ and $a_{m+1}^{\prime} \in A_{m+1}^{\prime}$ such that $\ell_{a_{1}^{\prime} \ldots a_{m+1}^{\prime}}^{a_{1} \ldots a_{m+1}}=\infty$. We can choose an infinite descending path such that $\ell_{a_{1}^{\prime} \ldots a_{m}^{\prime}}^{a_{1} \ldots a_{m}}$ is always infinite. The sets $B_{a_{1}^{\prime} \ldots a_{m}^{\prime}}^{a_{1} \ldots a_{m}}$ are not empty for any $m$, so that $B_{a_{1}^{\prime} a_{2}^{\prime} \ldots}^{a_{1} a_{2} \ldots}$ is a non-empty limiting set.

3.2. An extension theorem. We now give a criterion for a set function $\mu$ on $\Sigma^{\prime}$ to extend to a measure on $\Sigma$.

Theorem 3.2. Let $\Sigma^{\prime}$ be the semiring generated by the cylinder sets $B_{i, j}$ in (3.1), and let $\mu$ be an additive set function on $\Sigma^{\prime}$ with $\mu\left(\mathcal{S}_{\mathbb{N}}\right)<\infty$. We assume that for all $i \in \mathbb{N}$, and all $\varepsilon>0$, there exists a finite set $A \subset \mathbb{N}$ (that depends on $i$ and $\varepsilon$ ) such that

$$
\sum_{j \in A} \mu\left(B_{i, j}\right)>1-\varepsilon, \quad \text { and } \quad \sum_{j \in A} \mu\left(B_{j, i}\right)>1-\varepsilon .
$$

Then $\mu$ extends uniquely to a measure on $\left(\mathcal{S}_{\mathbb{N}}, \Sigma\right)$. 
If $\mu$ is symmetric with respect to the inversion of permutation, i.e. $\mu\left(B_{i, j}\right)=\mu\left(B_{j, i}\right)$, then the two conditions are equivalent to

$$
\sum_{j=1}^{\infty} \mu\left(B_{i, j}\right)=\mu\left(\mathcal{S}_{\mathbb{N}}\right)
$$

for any fixed $i$. Since $\mathcal{S}_{\mathbb{N}}=\cup_{j} B_{i, j}$, the condition amounts to $\sigma$-additivity for a restricted class of disjoint unions. Thus (3.8) is also a necessary condition.

Proof. It follows from the assumption of the theorem that for any $\varepsilon>0$, there exist finite sets $C_{1}, C_{2}, \ldots$ such that, for all $i$,

$$
\mu\left(\left\{\pi \in \mathcal{S}_{\mathbb{N}}: \pi^{-1}(i) \in C_{i} \text { and } \pi(i) \in C_{i}\right\}\right)>1-2^{-i-1} \varepsilon .
$$

Then for any $m$, we have

$$
\mu\left(B_{C_{1}^{\prime} \ldots C_{m}^{\prime}}^{C_{1} \ldots C_{m}}\right)>1-\frac{1}{2} \varepsilon
$$

We prove the following property, that is equivalent to $\sigma$-additivity in $\Sigma^{\prime}$. For any decreasing sequence $\left(G_{n}\right)$ of sets in $\Sigma^{\prime}$ such that $\mu\left(G_{n}\right)>\varepsilon$ for all $n$, we have $\lim _{n} G_{n} \neq \emptyset$. As we have observed above, $G_{n}$ can be written as

$$
G_{n}=B_{A_{n 1}^{\prime} \ldots A_{n m}^{\prime}}^{A_{n 1} \ldots A_{n m}}
$$

for some finite $m$ that depends on $n$. Without loss of generality, we can suppose that $m \geqslant n$ (choosing some sets to be $\mathbb{N}$ if necessary); we can actually take $m=n$ (by restricting to a subsequence if necessary). This allows to alleviate a bit the argument.

We have $G_{n} \cap G_{n+1}=G_{n+1}$. Using (3.3), we can choose the sets such that $A_{n i} \supset A_{n+1, i}$ and $A_{n i}^{\prime} \supset A_{n+1, i}^{\prime}$ for each $i$. The sets $A_{n i}, A_{n i}^{\prime}$ may be infinite. We therefore define the finite sets $D_{n i}=A_{n i} \cap C_{i}$ and $D_{n i}^{\prime}=A_{n i}^{\prime} \cap C_{i}$. Then

$$
\begin{aligned}
\mu\left(B_{D_{n 1}^{\prime} \ldots D_{n n}^{\prime}}^{D_{n 1} \ldots D_{n n}}\right) & =\mu\left(G_{n} \cap B_{C_{1} \ldots C_{n}}^{C_{1} \ldots C_{n}}\right) \\
& \geqslant \mu\left(G_{n}\right)-\mu\left(\left[B_{C_{1} \ldots C_{n}}^{C_{1} \ldots C_{n}}\right]^{\mathrm{c}}\right) \\
& >\frac{1}{2} \varepsilon .
\end{aligned}
$$

The sets $D_{n i}, D_{n i}^{\prime}$ are finite and decreasing for fixed $i$. Let

$$
D_{i}=\lim _{n} D_{n i}, \quad D_{i}^{\prime}=\lim _{n} D_{n i}^{\prime} .
$$

For any $k$, there exists $n$ large enough such that

$$
B_{D_{n 1}^{\prime} \ldots D_{n n}^{\prime}}^{D_{n 1} \ldots D_{n n}} \subset B_{D_{1}^{\prime} \ldots D_{k}^{\prime}}^{D_{1} \ldots D_{k}} \subset G_{k} .
$$

The set in the left side is not empty since it has positive measure. Thus the set in the middle is not empty for any $k$. By Lemma 3.1, its limit as $k \rightarrow \infty$ is not empty, which proves that $\lim _{k} G_{k} \neq \emptyset$.

Although Theorem 3.2 does not contain any reference to space, we see in the following section that the spatial structure provides a natural way for $(3.8)$ to be fulfilled in certain cases. 
3.3. Permutation cycles and probability measure. Here we discuss the connection between the considerations of the two previous subsections, and the spatial structure. The set of permutations where $i$ belongs to a cycle of length $n$ can be expressed as

$$
B_{i}^{(n)}=\bigcup_{j_{1}, \ldots, j_{n}} \bigcap_{i=1}^{n} B_{j_{i-1}, j_{i}}
$$

where $j_{1}, \ldots, j_{n}$ are distinct integers, and where we set $j_{0} \equiv j_{n}$. The union is countable and $B_{i}^{(n)}$ belongs to the $\sigma$-algebra $\Sigma$. The event where $i$ belongs to an infinite cycle is then

$$
B_{i}^{(\infty)}=\left[\bigcup_{n \geqslant 1} B_{i}^{(n)}\right]^{\mathrm{c}}
$$

and it also belongs to $\Sigma$. We can introduce the random variable $\ell_{i}$ for the length of the cycle that contains $i$. It can take the value $\infty$. Since $\ell_{i}^{-1}(\{n\})=B_{i}^{(n)}$ for all $n=1,2, \ldots, \infty$, we see that $\ell_{i}$ is measurable.

In general the probability distribution of $\ell_{i}$ depends on $i$. Thus we average over points in a large domain. Let $\boldsymbol{x} \subset \mathbb{R}^{d}$ be a countable set with no accumulation points (that is, if $\Lambda \subset \mathbb{R}^{d}$ is bounded, then $\boldsymbol{x} \cap \Lambda$ is finite). The elements $x_{1}, x_{2}, \ldots$ of $\boldsymbol{x}$ can be ordered according to their distance to the origin. More exactly, we suppose that for any cube $\Lambda$ centered at the origin, there exists $N$ such that $x_{i} \in \Lambda$ iff $i \leqslant N$. Let $V$ be the volume of $\Lambda$. We introduce the density of points in cycles of length between $m$ and $n$, by

$$
\varrho_{m, n}^{(\Lambda)}(\pi)=\frac{1}{V} \#\left\{i=1, \ldots, N: m \leqslant \ell_{i}(\pi) \leqslant n\right\} .
$$

This expression is of course very similar to Eq. (2.5) for the model in finite volume.

Next we define the relevant measure on $\mathcal{S}_{\mathbb{N}}$. Let $\mathcal{S}_{N}$ be the set of permutations that are trivial for indices larger than $N$ :

$$
\mathcal{S}_{N}=\left\{\pi \in \mathcal{S}_{\mathbb{N}}: \pi(i)=i \text { if } i>N\right\} .
$$

We define the finite volume probability of a set $B$ in the semiring $\Sigma^{\prime}$ by

$$
\nu_{\boldsymbol{x}}^{(\Lambda)}(B)=\frac{1}{Y\left(\boldsymbol{x}_{\Lambda}\right)} \sum_{\pi \in B \cap \mathcal{S}_{N}} \mathrm{e}^{-H\left(\boldsymbol{x}_{\Lambda}, \pi\right)} .
$$

Here, $\boldsymbol{x}_{\Lambda}=\boldsymbol{x} \cap \Lambda$. The Hamiltonian $H\left(\boldsymbol{x}_{\Lambda}, \pi\right)$ and the normalisation $Y\left(\boldsymbol{x}_{\Lambda}\right)$ are given by the same expression as in Section 2 .

The existence of the thermodynamic limit turns out to be difficult to establish. If $\boldsymbol{x}$ is a lattice such as $\mathbb{Z}^{d}$, or if $\boldsymbol{x}$ is the realisation of a translation invariant point process, we expect that $\nu_{\boldsymbol{x}}^{(\Lambda)}(B)$ converges as $V \rightarrow \infty$. We cannot prove such a strong statement, but it follows from Cantor's diagonal argument that there exists a subsequence $\left(V_{n}\right)$ of increasing volumes, such that $\nu_{\boldsymbol{x}}^{\left(\Lambda_{n}\right)}(B)$ converges for all $B \in \Sigma^{\prime}$. (Here, $\Lambda_{n}$ is the cube of volume $V_{n}$ centered at 0 .) Thus we have existence of a limiting set function, $\nu_{\boldsymbol{x}}$, but we cannot garantee its uniqueness.

If $\xi$ involves a cutoff, i.e. if $\mathrm{e}^{-\xi(x)}$ is zero for $x$ large enough, 3.8 is fulfiled and $\nu_{\boldsymbol{x}}$ extends to an infinite volume measure thanks to Theorem 3.2. But we cannot prove the criterion of the theorem for more general $\xi$, not even the Gaussian. It is certainly true, though.

For relevant choices of point processes and of permutation measures, we expect that $\lim _{V \rightarrow \infty} \varrho_{m, n}^{(\Lambda)}(\pi)$ exists for a.e. $\boldsymbol{x}$ and a.e. $\pi$. Let $\varrho_{m, n}$ denote the limiting random variable. 
It allows to define the expectation

$$
E\left(\varrho_{m, n}\right)=\int \mathrm{d} \mu(\boldsymbol{x}) \int \mathrm{d} \nu_{\boldsymbol{x}}(\pi) \varrho_{m, n}(\pi) .
$$

It would be interesting to obtain properties such as concentration of the distribution of $\varrho_{m, n}$. The simplest case should be the Poisson point process.

3.4. Finite vs infinite volume. The finite volume setting of Section 2 can be rephrased in the infinite volume setting as follows. Let $\mu^{(\Lambda, N)}$ be the point process on $\mathbb{R}^{d}$ such that

$$
\mu^{(\Lambda, N)}(\mathrm{d} \boldsymbol{x})= \begin{cases}\frac{Y(\boldsymbol{x})}{Z(\Lambda, N) N !} \mathrm{d} \boldsymbol{x} & \text { if } \boldsymbol{x} \subset \Lambda \text { and }|\boldsymbol{x}|=N, \\ 0 & \text { otherwise. }\end{cases}
$$

Next, let $\nu_{\boldsymbol{x}}^{(\Lambda)}$ be as in 3.20 . For $\Lambda_{1} \supset \Lambda_{2} \supset \Lambda_{3}$, let us consider the expectation

$$
E_{\Lambda_{1}, \Lambda_{2}, N}\left(\varrho_{m, n}^{\left(\Lambda_{3}\right)}\right)=\int \mathrm{d} \mu^{\left(\Lambda_{1}, N\right)}(\boldsymbol{x}) \int \mathrm{d} \nu_{\boldsymbol{x}}^{\left(\Lambda_{2}\right)}(\pi) \varrho_{m, n}^{\left(\Lambda_{3}\right)}
$$

This can be compared to Eqs 2.4 and 2.5. Namely, we have

$$
E_{\Lambda, N}\left(\varrho_{m, n}\right)=E_{\Lambda, \Lambda, N}\left(\varrho_{m, n}^{(\Lambda)}\right)
$$

It would be interesting to prove that the infinite volume limits in 3.23 can be taken separately. Precisely, we expect that

$$
\lim _{\Lambda \nearrow \mathbb{R}^{d}} E_{\Lambda, \rho|\Lambda|}\left(\boldsymbol{\varrho}_{m, n}\right)=\lim _{\Lambda_{3} \nearrow \mathbb{R}^{d}} \lim _{\Lambda_{2} \nearrow \mathbb{R}^{d}} \lim _{\Lambda_{1} \nearrow \mathbb{R}^{d}} E_{\Lambda_{1}, \Lambda_{2}, \rho\left|\Lambda_{1}\right|}\left(\varrho_{m, n}^{\left(\Lambda_{3}\right)}\right) .
$$

\section{A Regime Without infinite CyCles}

At low density the jumps are very much discouraged and the typical permutations resemble the identity permutation, up to few small cycles here and there. In this section we give a sufficient condition for the absence of infinite cycles. We consider the infinite volume framework. The condition has two parts: (a) a bound on the strength of the interactions; (b) in essence, that the points of $\boldsymbol{x}$ lie far apart compared to the decay length of $\mathrm{e}^{-\xi}$. Here, $B^{(\gamma)}$ denotes the set of permutations where the cycle $\gamma$ is present.

Theorem 4.1. Let $\boldsymbol{x} \subset \mathbb{R}^{d}$ be a countable set, and let $i$ be an integer. We assume the following conditions on interactions and on jump factors, respectively.

(a) There exists $0 \leqslant s<1$ such that for all cycles $\gamma=\left(j_{1}, \ldots, j_{n}\right) \subset \mathbb{N}$ long enough, with $j_{1}=i$, and all permutations $\pi \in B^{(\gamma)}$,

$$
\sum_{\substack{A \subset \mathbb{N} \\ A \cap \gamma \neq \emptyset}}\left|V\left(\left(x_{j}, x_{\pi(j)}\right)_{j \in A}\right)-V\left(\left(x_{j}, x_{\bar{\pi}(j)}\right)_{j \in A}\right)\right| \leqslant s \sum_{j=1}^{n} \xi\left(x_{j}-x_{j-1}\right),
$$

with $x_{0} \equiv x_{n}$, and where $\bar{\pi}$ is the permutation obtained from $\pi$ by removing $\gamma$, i.e.

$$
\bar{\pi}(j)= \begin{cases}\pi(j) & \text { if } j \neq j_{1}, \ldots, j_{n}, \\ j & \text { if } j=j_{k} \text { for some } k\end{cases}
$$

(b) With the same $s$ as in (a),

$$
\sum_{n \geqslant 1} \sum_{\substack{\gamma=\left(j_{1}, \ldots, j_{n}\right) \\ j_{1}=i}} \prod_{k=1}^{n} \mathrm{e}^{-(1-s) \xi\left(x_{j_{k}}-x_{j_{k-1}}\right)}<\infty
$$


Then we have

$$
\lim _{n \rightarrow \infty} \lim _{V \rightarrow \infty} \sum_{k \geqslant n} \nu_{\boldsymbol{x}}^{(\Lambda)}\left(B_{i}^{(k)}\right)=0 .
$$

The condition (a) is stated "for all cycles long enough", i.e. it must hold for all cycles of length $n \geqslant n_{0}$, for some fixed $n_{0}$ that depends on $i$ only. This weakening of the condition is useful; many points are involved, they cannot be too close, and $\sum \xi(\cdot)$ in the right side cannot be too small.

If $\nu_{\boldsymbol{x}}$ extends to a measure on $\Sigma$, then

$$
\lim _{n \rightarrow \infty} \lim _{V \rightarrow \infty} \sum_{k \geqslant n} \nu_{\boldsymbol{x}}^{(\Lambda)}\left(B_{i}^{(k)}\right)=\nu_{\boldsymbol{x}}\left(B_{i}^{(\infty)}\right),
$$

and the theorem states that $\nu_{\boldsymbol{x}}\left(B_{i}^{(\infty)}\right)=0$.

An open problem is to provide sufficient conditions on the Hamiltonian such that, in the finite volume framework, we have

$$
\lim _{M \rightarrow \infty} \lim _{V \rightarrow \infty} E_{\Lambda, \rho V}\left(\varrho_{M, \rho V}\right)=0 .
$$

Proof of Theorem 4.1. By definition,

$$
\nu_{\boldsymbol{x}}^{(\Lambda)}\left(B_{i}^{(k)}\right)=\frac{1}{Y\left(\boldsymbol{x}_{\Lambda}\right)} \sum_{\substack{\gamma=\left(j_{1}, \ldots, j_{k}\right) \\ j_{1}=i}} \sum_{\pi \in B_{N}^{(\gamma)}} \mathrm{e}^{\left.-\sum_{l=1}^{k} \xi\left(x_{i_{l}}-x_{i_{l}-1}\right)-\sum_{A \cap \gamma \neq \emptyset} V\left(\left(x_{j}, x_{\pi(j)}\right)\right)_{j \in A}\right)-H\left(\boldsymbol{x}_{\Lambda \backslash \gamma}, \pi\right)}
$$

with $B_{N}^{(\gamma)}=B^{(\gamma)} \cap \mathcal{S}_{N}$. By restricting the sum over permutations, we get a lower bound for the partition function:

$$
Y\left(\boldsymbol{x}_{\Lambda}\right) \geqslant \sum_{\pi \in B_{N}^{(\gamma)}} \exp \left\{-\sum_{A \cap \gamma \neq \emptyset} V\left(\left(x_{j}, x_{\bar{\pi}(j)}\right)_{j \in A}\right)-H\left(\boldsymbol{x}_{\Lambda}, \bar{\pi}\right)\right\} .
$$

Observe that $H\left(\boldsymbol{x}_{\Lambda \backslash \gamma}, \pi\right)=H\left(\boldsymbol{x}_{\Lambda}, \bar{\pi}\right)$. Combining with the two conditions of the theorem, we obtain

$$
\sum_{k \geqslant n} \nu_{\boldsymbol{x}}^{(\Lambda)}\left(B_{i}^{(k)}\right) \leqslant \sum_{k \geqslant n} \sum_{\substack{\gamma=\left(j_{1}, \ldots, j_{k}\right) \\ j_{1}=i}} \prod_{l=1}^{n} \mathrm{e}^{-(1-s) \xi\left(x_{j_{l}}-x_{j_{l-1}}\right)} .
$$

The right side does not depend on $\Lambda$, and it vanishes in the limit $n \rightarrow \infty$.

\section{THE ONE-BODY MODEL}

5.1. Occurrence of infinite cycles. The occurrence of infinite cycles can be proved in a large class of models with one-body Hamiltonians, with the critical density being exactly known! Here the domain $\Lambda$ is the cubic box of size $L$, volume $V=L^{d}$. We fix the density $\rho$ of points, that is, we take $N=\rho V$.

Recall the definition 2.5 for the random variable $\varrho_{m, n}$ that gives the density of points in cycles of length between $m$ and $n$. We consider the Hamiltonian

$$
H(\boldsymbol{x}, \pi)=\sum_{i=1}^{N} \xi_{\Lambda}\left(x_{i}-x_{\pi(i)}\right)
$$

where the function $\xi_{\Lambda}$ is a slight modification of the function $\xi$, defined by the relation

$$
\mathrm{e}^{-\xi_{\Lambda}(x)}=\sum_{y \in \mathbb{Z}^{d}} \mathrm{e}^{-\xi(x-L y)}
$$


Our conditions on $\xi$ ensure that the latter sum is finite, and that $\xi_{\Lambda}$ converges pointwise to $\xi$ as $V \rightarrow \infty$. This technical modification should not be necessary, but it allows to simplify the proof of Theorem 5.1 below. In essence, this amounts to choosing periodic boundary conditions.

Let $C=\int \mathrm{e}^{-\xi}$. We suppose that the Fourier transform of $\mathrm{e}^{-\xi(x)}$ is nonnegative, and we denote it $C \mathrm{e}^{-\varepsilon(k)}:$ For $k \in \mathbb{R}^{d}$,

$$
C \mathrm{e}^{-\varepsilon(k)}=\int_{\mathbb{R}^{d}} \mathrm{e}^{-2 \pi \mathrm{i} k x} \mathrm{e}^{-\xi(x)} \mathrm{d} x
$$

The "dispersion relation" $\varepsilon(k)$ always satisfies

- $\varepsilon(0)=0 ; \varepsilon(k)>a|k|^{2}$ for small $k$ (indeed, the Laplacian of $\mathrm{e}^{-\varepsilon(k)}$ would otherwise be zero at $k=0$; then $\int|x|^{2} \mathrm{e}^{-\xi(x)} \mathrm{d} x=0$, which is absurd).

- $\varepsilon(k)>0$ uniformly in $k$ away from zero;

- $\int \mathrm{e}^{-\varepsilon(k)} \mathrm{d} k=C^{-1}<\infty$.

Among many examples of functions that satisfy the conditions above, let us mention several important cases.

(1) The Gaussian:

$$
\mathrm{e}^{-\xi(x)}=\mathrm{e}^{-|x|^{2} / 4 \beta}, \quad \varepsilon(k)=4 \pi^{2} \beta|k|^{2}, \quad C=(4 \pi \beta)^{d / 2} .
$$

(2) In dimension $d=3$, the exponential:

$$
\mathrm{e}^{-\xi(x)}=\mathrm{e}^{-|x| / \beta}, \quad \varepsilon(k)=2 \log \left[1+(2 \pi \beta|k|)^{2}\right], \quad C=8 \pi / \beta^{3} .
$$

(3) In $d=3$, a sufficient condition for positive Fourier transform is that

$$
\frac{1}{r}\left(\mathrm{e}^{-\xi(r)}\right)^{\prime \prime}
$$

be monotone decreasing; here, $\xi$ depends on $r=|x|$ only. See e.g. 13 and references therein. Thus there exist functions $\mathrm{e}^{-\xi}$ with compact support and positive Fourier transform.

(4) In $d=1$,

$$
\mathrm{e}^{-\xi(x)}=(|x|+1)^{-3 / 2} \text {. }
$$

Its Fourier transform is positive by Lemma B.1. It can be checked that $\varepsilon(k) \sim|k|^{1 / 2}$ for small $k$, so that its critical density is finite.

We define the critical density by

$$
\rho_{\mathrm{c}}=\int_{\mathbb{R}^{d}} \frac{\mathrm{d} k}{\mathrm{e}^{\varepsilon(k)}-1} .
$$

The critical density is finite for $d \geqslant 3$, but it can be infinite in $d=1,2$. The following theorem claims that infinite cycles are present for $\rho>\rho_{\mathrm{c}}$ only, and that they are macroscopic. It extends results of Sütö for the ideal Bose gas [26], which corresponds to the Gaussian case $\xi(x) \sim|x|^{2}$. The theorem also applies when $\rho_{\mathrm{c}}=\infty$. 
Theorem 5.1. Let $\xi$ satisfy the assumptions above. Then for all $0<a<b<1$, and all $s \geqslant 0$,

$$
\begin{aligned}
& \text { (a) } \lim _{V \rightarrow \infty} E_{\Lambda, \rho V}\left(\varrho_{1, V^{a}}\right)= \begin{cases}\rho & \text { if } \rho \leqslant \rho_{\mathrm{c}} \\
\rho_{\mathrm{c}} & \text { if } \rho \geqslant \rho_{\mathrm{c}}\end{cases}
\end{aligned}
$$

The rest of this section is devoted to the proof of this theorem.

5.2. Fourier representation for spatial permutations. The first step is to reformulate the problem in the Fourier space. In the Gaussian case, $\xi(x)=|x|^{2}$, this is traditionally done using the Feynman-Kac formula and unitary transformations of Hilbert spaces. There is no Feynman-Kac formula for our more general setting, but we can directly use the Fourier transform. This actually simplifies the situation.

Here and in the sequel, we use the definition (5.3) for the Fourier transform. Let $\Lambda$ be the unit cube, i.e. we fix the length to $L=1$. For $f \in L^{1}\left(\mathbb{R}^{d}\right)$, let $f_{\Lambda}(x)=\sum_{y \in \mathbb{Z}^{d}} f(x+y)$; notice that $f_{\Lambda} \in L^{1}(\Lambda)$.

Lemma 5.2. Let $f \in L^{1}\left(\mathbb{R}^{d}\right)$. For all $n=1,2, \ldots$, we have

$$
\int_{\Lambda^{n}} \mathrm{~d} x_{1} \ldots \mathrm{d} x_{n} \prod_{i=1}^{n} f_{\Lambda}\left(x_{i}-x_{i-1}\right)=\sum_{k \in \mathbb{Z}^{d}} \widehat{f}(k)^{n} .
$$

(By definition, $x_{0}=x_{n}$.)

Proof. The $n$-th convolution of the function $f_{\Lambda}$ with itself satisfies

$$
\left(f_{\Lambda}^{* n}\right)(x)=\int \mathrm{d} x_{1} \ldots \mathrm{d} x_{n-1} f_{\Lambda}\left(x_{1}\right) f_{\Lambda}\left(x_{2}-x_{1}\right) \ldots f_{\Lambda}\left(x-x_{n-1}\right)
$$

The product $\prod f_{\Lambda}\left(x_{i}-x_{i-1}\right)$ is translation invariant, so that

$$
\begin{aligned}
\int_{\Lambda^{n}} \mathrm{~d} x_{1} \ldots \mathrm{d} x_{n} \prod_{i=1}^{n} f_{\Lambda}\left(x_{i}-x_{i-1}\right) & =\int_{\Lambda^{n}} \mathrm{~d} x_{1} \ldots \mathrm{d} x_{n} f_{\Lambda}\left(x_{1}\right) f_{\Lambda}\left(x_{2}-x_{1}\right) f_{\Lambda}\left(-x_{n-1}\right) \\
& =f_{\Lambda}^{* n}(0) \\
& =\sum_{k \in \mathbb{Z}^{d}} \widehat{f_{\Lambda}^{* n}}(k) \\
& =\sum_{k \in \mathbb{Z}^{d}} \widehat{f}(k)^{n} .
\end{aligned}
$$

The hat symbol in the third line denotes the $L^{2}(\Lambda)$-Fourier transform; but in the fourth line, it denotes the $L^{2}\left(\mathbb{R}^{d}\right)$-Fourier transform.

We will use a corollary of this lemma. 
Corollary 5.3. Let $\Lambda$ be a cube of size $L, \Lambda^{*}=\left(\frac{1}{L} \mathbb{Z}\right)^{d}$ be the dual space, and let $f_{\Lambda}(x)=$ $\sum_{y \in \mathbb{Z}^{d}} f(x+L y)$. Then for any permutation $\pi \in \mathcal{S}_{N}$ we have:

$$
\int_{\Lambda^{N}} \mathrm{~d} x_{1} \ldots \mathrm{d} x_{N} \prod_{i=1}^{N} f_{\Lambda}\left(x_{i}-x_{\pi(i)}\right)=\sum_{k_{1}, \ldots, k_{N} \in \Lambda^{*}} \prod_{i=1}^{N} \delta_{k_{i}, k_{\pi(i)}} \prod_{i=1}^{N} \widehat{f}\left(k_{i}\right) .
$$

Proof. It is enough to consider the case $L=1$, the general case can be obtained by scaling. The multiple integral factorises according to the cycles of the permutation. Using Lemma 5.2 for each cycle, we get the result.

We are now in position to reformulate the problem in the Fourier space. By Corollary 5.3 , we have the relation

$$
E_{\Lambda, N}(\theta)=\frac{1}{Z^{\prime}(\Lambda, N) N !} \sum_{\pi \in \mathcal{S}_{N}} \theta(\pi) \sum_{k_{1}, \ldots, k_{N} \in \Lambda^{*}} \mathrm{e}^{-\sum_{i=1}^{N} \varepsilon\left(k_{i}\right)} \prod_{i=1}^{N} \delta_{k_{i}, k_{\pi(i)}}
$$

with $Z^{\prime}(\Lambda, N)=C^{-N} Z(\Lambda, N)$.

A simpler expression than (5.11) is available for random variables invariant under transposition, i.e. $\theta \in \mathcal{R}_{N}$. To obtain it, we introduce the set $\mathcal{N}_{\Lambda}$ of "occupation numbers" on $\Lambda^{*}$. An element $\boldsymbol{n} \in \mathcal{N}_{\Lambda}$ is a sequence of integers $\boldsymbol{n}=\left(n_{k}\right), n_{k}=0,1,2, \ldots$, indexed by $k \in \Lambda^{*}$. We denote by $\mathcal{N}_{\Lambda, N}$ the set of occupation numbers with total number $N$ :

$$
\mathcal{N}_{\Lambda, N}=\left\{\boldsymbol{n} \in \mathcal{N}_{\Lambda}: \sum_{k \in \Lambda^{*}} n_{k}=N\right\}
$$

Let $\boldsymbol{k}=\left(k_{1}, \ldots, k_{N}\right)$ be an $N$-tuple of elements of $\Lambda^{*}$. We can assign an element $\boldsymbol{n}=\boldsymbol{n}(\boldsymbol{k})$ in $\mathcal{N}_{\Lambda, N}$ by defining, for each $k \in \Lambda^{*}$,

$$
n_{k}=\#\left\{i=1, \ldots, N: k_{i}=k\right\} .
$$

In other words, $n_{k}$ is the number of occurrences of the vector $k$ in $\boldsymbol{k}$. The map $\boldsymbol{k} \mapsto \boldsymbol{n}(\boldsymbol{k})$ is onto but not one-to-one; the number of $\boldsymbol{k}$ 's that are sent to a given $\boldsymbol{n}$ is equal to

$$
N ! / \prod_{k \in \Lambda^{*}} n_{k} !
$$

We now introduce probabilities for Fourier modes, permutations, and occupation numbers. The sample space is $\left(\Lambda^{*}\right)^{N} \times \mathcal{S}_{N}$; it is discrete, and we consider the discrete $\sigma$ algebra. When taking probabilities, we write $\boldsymbol{k} \times \pi$ for $\{(\boldsymbol{k}, \pi)\} ; \boldsymbol{k}$ for $\{\boldsymbol{k}\} \times \mathcal{S}_{N} ; \boldsymbol{n}$ for $\{\boldsymbol{k}: \boldsymbol{n}(\boldsymbol{k})=\boldsymbol{n}\} \times \mathcal{S}_{N}$ and $\pi$ for $\left(\Lambda^{*}\right)^{N} \times\{\pi\}$. We define the probability $P_{\Lambda, N}$ by

$$
P_{\Lambda, N}(\boldsymbol{k} \times \pi)= \begin{cases}\frac{1}{Z^{\prime}(\Lambda, N) N !} \mathrm{e}^{-\sum_{i=1}^{N} \varepsilon\left(k_{i}\right)} & \text { if } k_{i}=k_{\pi(i)} \text { for all } i, \\ 0 & \text { otherwise. }\end{cases}
$$

Here, $\boldsymbol{k}=\left(k_{1}, \ldots, k_{N}\right)$. Summing over permutations, we get

$$
P_{\Lambda, N}(\boldsymbol{k})=\frac{1}{Z^{\prime}(\Lambda, N) N !} \mathrm{e}^{-\sum_{k \in \Lambda^{*}} \varepsilon(k) n_{k}} \prod_{k \in \Lambda^{*}} n_{k} !
$$

with $\left(n_{k}\right)=\boldsymbol{n}(\boldsymbol{k})$. Finally, summing over Fourier modes that are compatible with occupation numbers yields

$$
P_{\Lambda, N}(\boldsymbol{n})=\frac{1}{Z^{\prime}(\Lambda, N)} \mathrm{e}^{-\sum_{k \in \Lambda^{*} \varepsilon(k) n_{k}}}
$$


It follows that the partition function $Z^{\prime}(\Lambda, N)$ can be expressed using occupation numbers as

$$
Z^{\prime}(\Lambda, N)=\sum_{\boldsymbol{n} \in \mathcal{N}_{\Lambda, N}} \mathrm{e}^{-\sum_{k \in \Lambda^{*}} \varepsilon(k) n_{k}}
$$

These definitions allow to express $E_{\Lambda, N}(\theta)$ in an illuminating form.

Lemma 5.4. For all $\theta \in \mathcal{R}_{N}$,

$$
E_{\Lambda, N}(\theta)=\sum_{\boldsymbol{n} \in \mathcal{N}_{\Lambda, N}} P_{\Lambda, N}(\boldsymbol{n}) \sum_{\pi \in \mathcal{S}_{N}} \theta(\pi) P_{\Lambda, N}(\pi \mid \boldsymbol{k})
$$

with $\boldsymbol{k}$ any $N$-tuple of Fourier modes that is compatible with $\boldsymbol{n}$, i.e. such that $\boldsymbol{n}(\boldsymbol{k})=\boldsymbol{n}$.

Proof. Using the definitions (5.14) $-(5.16)$, the expectation (5.11) of $\theta$ can be written as

$$
\begin{aligned}
E_{\Lambda, N}(\theta) & =\sum_{\boldsymbol{k} \in\left(\Lambda^{*}\right)^{N}} \sum_{\pi \in \mathcal{S}_{N}} \theta(\pi) P_{\Lambda, N}(\boldsymbol{k} \times \pi) \\
& =\sum_{\boldsymbol{k} \in\left(\Lambda^{*}\right)^{N}} P_{\Lambda, N}(\boldsymbol{k}) \sum_{\pi \in \mathcal{S}_{N}} \theta(\pi) P_{\Lambda, N}(\pi \mid \boldsymbol{k}) .
\end{aligned}
$$

The latter sum does not depend on the ordering of the $k_{i}$ 's - it depends only on occupation numbers (notice that $P_{\Lambda, N}(\pi \mid \sigma(\boldsymbol{k}))=P_{\Lambda, N}\left(\sigma^{-1} \pi \sigma \mid \boldsymbol{k}\right)$ for all $\left.\sigma \in \mathcal{S}_{N}\right)$. The lemma follows from (5.16).

\section{Lemma 5.5.}

$$
\sum_{\pi \in \mathcal{S}_{N}} \varrho_{m, n}(\pi) P_{\Lambda, N}(\pi \mid \boldsymbol{k})=\frac{1}{V} \sum_{k \in \Lambda^{*}} \begin{cases}n-m+1 & \text { if } 1 \leqslant m \leqslant n \leqslant n_{k} \\ n_{k}-m+1 & \text { if } 1 \leqslant m \leqslant n_{k} \leqslant n \\ 0 & \text { if } n_{k}<a \leqslant b .\end{cases}
$$

Proof. It follows from (5.14) that, given $\boldsymbol{k}$, permutations are uniformly distributed (over compatible permutations). That is,

$$
P_{\Lambda, N}(\pi \mid \boldsymbol{k})= \begin{cases}1 / \prod_{k \in \Lambda^{*}} n_{k} ! & \text { if } k_{\pi(i)}=k_{i} \text { for all } i \\ 0 & \text { otherwise. }\end{cases}
$$

Given $\boldsymbol{k}=\left(k_{1}, \ldots, k_{N}\right)$, a permutation $\pi$ that leaves it invariant can be decomposed into a collection $\left(\pi_{k}\right), \pi_{k} \in \mathcal{S}_{n_{k}}$, of permutations for each Fourier mode, namely

$$
\sum_{\pi \in \mathcal{S}_{N}} \varrho_{m, n}(\pi) P_{\Lambda, N}(\pi \mid \boldsymbol{k})=\left(\prod_{k \in \Lambda^{*}} \sum_{\pi_{k} \in \mathcal{S}_{n_{k}}} \frac{1}{n_{k} !}\right) \varrho_{m, n}\left(\left(\pi_{k}\right)_{k \in \Lambda^{*}}\right) .
$$

In addition, we have

$$
\varrho_{m, n}\left(\left(\pi_{k}\right)_{k \in \Lambda^{*}}\right)=\frac{1}{V} \sum_{k \in \Lambda^{*}} N_{m, n}\left(\pi_{k}\right),
$$

with $N_{m, n}(\pi)$ being the number of indices that belong to cycles of length between $m$ and $n$. We obtain

$$
\sum_{\pi \in \mathcal{S}_{N}} \varrho_{m, n}(\pi) P_{\Lambda, N}(\pi \mid \boldsymbol{k})=\frac{1}{V} \sum_{k \in \Lambda^{*}} \frac{1}{n_{k} !} \sum_{\pi_{k} \in \mathcal{S}_{n_{k}}} N_{m, n}\left(\pi_{k}\right)
$$


We see that modes have been decoupled; further, we only need to average $N_{m, n}(\pi)$ over uniform permutations. One easily checks that the probability for an index to belong to a cycle of length $\ell$, with $N$ indices, is equal to $1 / N$ for any $\ell$. Then

$$
\frac{1}{N !} \sum_{\pi \in \mathcal{S}_{N}} N_{m, n}(\pi)=n-m+1
$$

for integers $1 \leqslant m \leqslant n \leqslant N$. The lemma follows.

We have all elements for the proof of Theorem 5.1 .

Proof of Theorem 5.1. We introduce a set $A_{\eta}$ of occupation numbers that is typical. With $\rho_{0}=\max \left(0, \rho-\rho_{\mathrm{c}}\right)$, let

$$
A_{\eta}=\left\{\boldsymbol{n} \in \mathcal{N}_{\Lambda, N}:\left|\frac{n_{0}}{V}-\rho_{0}\right|<\eta ; \sum_{0<|k|<V^{-\eta}} n_{k}<\eta V ; n_{k}<V^{3 \eta} \text { for all }|k| \geqslant V^{-\eta}\right\} \text {. }
$$

Notice that, for any $\boldsymbol{n} \in A_{\eta}$,

$$
\min \left(\rho, \rho_{\mathrm{c}}\right) V-2 \eta V \leqslant \sum_{|k| \geqslant V^{-\eta}} n_{k} \leqslant \min \left(\rho, \rho_{\mathrm{c}}\right) V+\eta V
$$

It is proved in Proposition A.2 that $P_{\Lambda, N}\left(A_{\eta}\right) \rightarrow 1$ as $V, N \rightarrow \infty$, for all $\eta>0$. Together with Lemmas 5.4 and 5.5, we obtain, with $N=\rho V$,

$$
\lim _{V \rightarrow \infty} E_{\Lambda, \rho V}\left(\varrho_{m, n}\right)=\lim _{V \rightarrow \infty} \sum_{\boldsymbol{n} \in A_{\eta}} P_{\Lambda, N}(\boldsymbol{n})\left[\varrho_{m, n}^{(0)}(\boldsymbol{n})+\boldsymbol{\varrho}_{m, n}^{(1)}(\boldsymbol{n})+\varrho_{m, n}^{(2)}(\boldsymbol{n})\right] .
$$

We partitioned $\Lambda^{*}$ into the disjoint union of $M^{(0)}=\{0\}, M^{(1)}=\left\{k: 0<|k|<V^{-\eta}\right\}$, and $M^{(2)}=\left\{k:|k| \geqslant V^{-\eta}\right\}$, and we introduced

$$
\boldsymbol{\varrho}_{m, n}^{(i)}(\boldsymbol{n})=\frac{1}{V} \sum_{k \in M^{(i)}}\left[(n-m+1) \chi_{[n, \infty)}\left(n_{k}\right)+\left(n_{k}-m+1\right) \chi_{[m, n)}\left(n_{k}\right)\right] .
$$

First, we note that $\varrho_{m, n}^{(1)}(\boldsymbol{n})<\eta$ for all $m, n$, so this term does not contribute in 5.26$)$. Next, assuming that $3 \eta<a<b$, we have

$$
\min \left(\rho, \rho_{\mathrm{c}}\right)-2 \eta \leqslant \varrho_{1, V^{a}}^{(2)}(\boldsymbol{n})=\frac{1}{V} \sum_{|k| \geqslant V^{-\eta}} n_{k} \leqslant \min \left(\rho, \rho_{\mathrm{c}}\right)+\eta
$$

We used (5.25). In addition, we observe that $\boldsymbol{\varrho}_{V^{a}, V^{b}}^{(2)}(\boldsymbol{n})$ and $\boldsymbol{\varrho}_{V^{b}, s V}^{(2)}(\boldsymbol{n})$ are zero for $\boldsymbol{n} \in A_{\eta}$. Let us turn to $\varrho_{m, n}^{(0)}$. We have

$$
\varrho_{1, V^{a}}^{(0)}(\boldsymbol{n})+\varrho_{V^{a}, V^{b}}^{(0)}(\boldsymbol{n})<V^{b-1}
$$

so these terms do not contribute in 5.26 . Finally, we have

$$
\varrho_{V^{b}, s V}^{(0)}(\boldsymbol{n})= \begin{cases}0 & \text { if } \rho \leqslant \rho_{\mathrm{c}}, \\ \frac{n_{0}}{V}-\frac{V^{b}+1}{V} & \text { if } \rho \geqslant \rho_{\mathrm{c}} \text { and } n_{0} \leqslant s V, \\ s-\frac{V^{b}+1}{V} & \text { if } \rho \geqslant \rho_{\mathrm{c}} \text { and } n_{0} \geqslant s V .\end{cases}
$$

Inserting these informations in 5.26 yields the theorem. 


\section{The Quantum Bose gas}

Einstein understood in 1925 that non-interacting bosons undergo a phase transition where a single particle state becomes macroscopically occupied. Ever since the Bose gas has stirred the interest of physicists and mathematical physicists. The first question that theoreticians needed to resolve was whether Bose-Einstein condensation also occurs in interacting systems, and what is the order parameter. Feynman introduced in 1953 what is now refered to as the Feynman-Kac representation [9]. It involves "space-time" trajectories and permutations, and Feynman emphasised the rôle played by long cycles. The correct order parameter, called "off-diagonal long-range order", was proposed shortly afterwards by Penrose and Onsager 23. But cycles did not disappear, and it was suggested in 1987 that winding cycles are related to superfluidity [24. Sütő made precise the notion of infinite cycles and he showed that it is equivalent to Bose-Einstein condensation in the ideal gas [25, 26]. An exact formula made this relation more explicit [27]. Recently, Boland and Pulé obtained puzzling results for the infinite-hopping Bose-Hubbard model [5]: Infinite cycles are present iff there is Bose-Einstein condensation, but the density of particles in infinite cycles is strictly greater than that in the condensate.

There are many open questions, of interest to physicists and mathematicians. The relationship between Bose-Einstein condensation, superfluidity, and infinite cycles, has yet to be clarified. The ideal gas can condense (if $d \geqslant 3$ ) but it is never superfluid. Interacting bosons in one or two dimensions do not condense but they may be superfluid. Infinite cycles seem related to Bose-Einstein condensation. It was argued in [28], however, that infinite cycles may be present in a solid at low temperature, so they do not automatically imply the existence of a condensate (nor a superfluid). On the other hand, it is expected that interacting bosons in three dimensions have a transition to a Bose condensate and a superfluid, and that this transition takes place at the same critical temperature. We also expect the critical temperature for infinite cycles to be the same. It is therefore of physical relevance to consider infinite cycles in the Feynman-Kac representation of the Bose gas. We discuss in Subsection 6.1 the relation between the Bose gas and models of spatial permutations. The latter should help us understand the effects of interactions on the critical temperature. This is discussed in Subsection 6.2.

The Bose gas has also been the object of many interesting mathematical studies. We only refer to [30] for a discussion of Bogoliubov theory and related work, and to [19] for remarkable results on the ground state of the interacting gas.

6.1. Feynman-Kac representation of the Bose gas. The state space for a system of $N$ identical bosons in a cube $\Lambda \subset \mathbb{R}^{d}$ (size $L$, volume $V=L^{d}$ ) is the Hilbert space $L_{\text {sym }}^{2}\left(\Lambda^{N}\right)$ of complex square-integrable symmetric functions on $\Lambda^{N}$. The Hamiltonian is the Schrödinger operator

$$
H=-\sum_{i=1}^{N} \Delta_{i}+\sum_{1 \leqslant i, j<N} U\left(x_{i}-x_{j}\right)
$$

Here, $\Delta_{i}$ denotes the $d$-dimensional Laplacian for the $i$-th variable, and $U\left(x_{i}-x_{j}\right)$ is a multiplication operator that represents the interaction between particles $i$ and $j$. We choose the self-adjoint extension for periodic boundary conditions.

In order to get cycles, and following [25], we work in the Hilbert space $L^{2}\left(\Lambda^{N}\right)$ and we consider the unitary representation of $\mathcal{S}_{N}$. That is, let $U_{\pi}$ denote the unitary operator

$$
U_{\pi} f\left(x_{1}, \ldots, x_{N}\right)=f\left(x_{\pi(1)}, \ldots, x_{\pi(N)}\right) .
$$


If $\theta$ is a function of permutations, we can define its expectation by

$$
\langle\theta\rangle_{\Lambda, N}=\frac{1}{Z(\Lambda, N) N !} \sum_{\pi \in \mathcal{S}_{N}} \theta(\pi) \operatorname{Tr} U_{\pi} \mathrm{e}^{-\beta H} .
$$

The Feynman-Kac formula expresses the operator $\mathrm{e}^{-\beta H}$ as an integral operator, whose kernel is given by

$$
\begin{aligned}
K\left(x_{1}, \ldots, x_{N} ; y_{1}, \ldots, y_{N}\right)=\int \mathrm{d} W_{x_{1} y_{1}}^{2 \beta}\left(\omega_{1}\right) \ldots \int \mathrm{d} W_{x_{N} y_{N}}^{2 \beta}\left(\omega_{N}\right) \\
\exp \left\{-\sum_{1 \leqslant i<j \leqslant N} \int_{0}^{2 \beta} U\left(\omega_{i}(s)-\omega_{j}(s)\right) \mathrm{d} s\right\} .
\end{aligned}
$$

Here, the Wiener measure $W_{x y}^{\beta}$ describes a Brownian bridge between $x$ and $y$ in time $\beta$, with periodic boundary conditions. We refer to [12] for an excellent introduction to the subject. We have

$$
\int \mathrm{d} W_{x y}^{\beta}(\omega)=\sum_{z \in \mathbb{Z}^{d}} g_{\beta}(x-y+L z) \equiv g_{\beta}^{(\Lambda)}(x-y)
$$

where $g_{\beta}$ denotes the normalised Gaussian function

$$
g_{\beta}(x)=\frac{1}{(2 \pi \beta)^{d / 2}} \mathrm{e}^{-|x|^{2} / 2 \beta} .
$$

The sum over $z$ in 6.5 accounts for periodic boundary conditions. For large $L$ the functions $g_{\beta}^{(\Lambda)}$ and $g_{\beta}$ are almost identical. If $f$ is a function $\mathbb{R}^{d n} \rightarrow \mathbb{R}$, and $0<t_{1}<\cdots<$ $t_{n}<\beta$ are ordered "times", we have

$$
\begin{aligned}
\int \mathrm{d} W_{x y}^{\beta}(\omega) f\left(\omega\left(t_{1}\right), \ldots, \omega\left(t_{n}\right)\right) & =\sum_{z \in \mathbb{Z}^{d}} \int_{\mathbb{R}^{d n}} \mathrm{~d} x_{1} \ldots \mathrm{d} x_{n} \\
& g_{t_{1}}^{(\Lambda)}\left(x-x_{1}\right) g_{t_{2}-t_{1}}^{(\Lambda)}\left(x_{2}-x_{1}\right) \ldots g_{\beta-t_{n}}^{(\Lambda)}\left(y-x_{n}\right) f\left(x_{1}, \ldots, x_{n}\right) .
\end{aligned}
$$

By the Feynman-Kac formula, the partition function is

$$
Z(\Lambda, N)=\operatorname{Tr}_{L_{\text {sym }}^{2}\left(\Lambda^{N}\right)} \mathrm{e}^{-\beta H}=\frac{1}{N !} \sum_{\pi \in \mathcal{S}_{N}} \int_{\Lambda^{N}} \mathrm{~d} x_{1} \ldots \mathrm{d} x_{N} K\left(x_{1}, \ldots, x_{N} ; x_{\pi(1)}, \ldots, x_{\pi(N)}\right) .
$$

The sum over permutations is present because we work in the symmetric subspace. The expectation of observables on permutations can be expressed as

$$
\langle\theta\rangle_{\Lambda, N}=\frac{1}{Z(\Lambda, N) N !} \sum_{\pi \in \mathcal{S}_{N}} \theta(\pi) \int_{\Lambda^{N}} \mathrm{~d} x_{1} \ldots \mathrm{d} x_{N} K\left(x_{1}, \ldots, x_{N} ; x_{\pi(1)}, \ldots, x_{\pi(N)}\right) .
$$

The connection to the model of spatial permutations is immediate in absence of interactions $(U(x) \equiv 0):\langle\theta\rangle_{\Lambda, N}=E_{\Lambda, N}(\theta)$ with $\xi(x)=|x|^{2} / 4 \beta, H^{(k)}=0$ for $k \geqslant 2$, and $G(\boldsymbol{x}) \equiv 0$. 
6.2. Discussion: Relevant interactions for spatial permutations. The models of spatial random permutations retain some of the features of the quantum Bose gas in the Feynman-Kac representation, but not all of them. The interactions between quantum particles translate into many-body interactions for permutations. However, an expansion reveals that to lowest order the interaction is two-body; precisely, the interaction between jumps $x \mapsto y$ and $x^{\prime} \mapsto y^{\prime}$ is given by

$$
V\left(x, y, x^{\prime}, y^{\prime}\right)=\int\left[1-\mathrm{e}^{-\frac{1}{4} \int_{0}^{4 \beta} U(\omega(s)) \mathrm{d} s}\right] \mathrm{d} \widehat{W}_{x-x^{\prime}, y-y^{\prime}}^{4 \beta}(\omega)
$$

for $x \neq y$. Here, $\widehat{W}_{x, y}^{t}=g_{t}^{-1}(x-y) W_{x, y}^{t}$ is a normalised Wiener measure, $\int \mathrm{d} \widehat{W}_{x, y}^{t}=1$. If $U$ consists of a hard-core potential of radius $a$, we notice that $V\left(x, y, x^{\prime}, y^{\prime}\right)$ is equal to the probability that a Brownian bridge, from $x-x^{\prime}$ to $y-y^{\prime}$, intersects the ball of radius $a$ centered at 0 .

The computation of (6.10) can be found in [29]; it is partly justified by a cluster expansion, although a rigorous result is still lacking. We expect that the critical temperature for the occurrence of infinite cycles is the same for $\langle\cdot\rangle_{\Lambda, N}$ and $E_{\Lambda, N}(\cdot)$ to lowest order in the strength of the interaction potential.

An important question about Bose systems concerns the effect of interactions on the critical temperature. Over the years physicists gave several, conflicting answers. But a consensus has recently emerged in the physics literature, mostly from path-integral MonteCarlo numerical studies. The critical temperature $T_{\mathrm{c}}^{(a)}$, as a function of the "scattering length" $a$ of the interaction potential (see e.g. [19] for the definition), behaves in three dimensions as

$$
\frac{T_{\mathrm{c}}^{(a)}-T_{\mathrm{c}}^{(0)}}{T_{\mathrm{c}}^{(0)}}=c \rho^{1 / 3} a+o\left(\rho^{1 / 3} a\right),
$$

with $c \approx 1.3$. See [2, 14, 15, 20] and references therein. The model of spatial random permutations should give the exact correction to the critical temperature, i.e. the correct constant $c$ in (6.11).

It is worth discussing the effects of interactions on spatial permutations in detail. They both modify the point process and the measure on permutations. Let $\mu^{(a)}$ denote the point process for an interaction potential of scattering length $a$, and $\nu_{\boldsymbol{x}}^{(a)}$ the measure on permutations. We can assign different parameters to these two measures, so as to have the expectation

$$
E^{\left(a, a^{\prime}\right)}(\theta)=\int \mathrm{d} \mu^{(a)}(\boldsymbol{x}) \int \mathrm{d} \nu_{\boldsymbol{x}}^{\left(a^{\prime}\right)}(\pi) \theta(\pi) .
$$

Let us fix the particle density, and let $T_{\mathrm{c}}^{\left(a, a^{\prime}\right)}$ denote the critical temperature for the occurrence of infinite cycles. Of course, $T_{\mathrm{c}}^{(a)}=T_{\mathrm{c}}^{(a, a)}$. For small $a$ we should have

$$
\frac{T_{\mathrm{c}}^{(a)}-T_{\mathrm{c}}^{(0)}}{T_{\mathrm{c}}^{(0)}}=\frac{T_{\mathrm{c}}^{(a, 0)}-T_{\mathrm{c}}^{(0)}}{T_{\mathrm{c}}^{(0)}}+\frac{T_{\mathrm{c}}^{(0, a)}-T_{\mathrm{c}}^{(0)}}{T_{\mathrm{c}}^{(0)}}+o(a) .
$$

The first question is whether both corrections are linear in $a$; if not, we could happily dismiss one. This formula may be useful in numerical simulations. The second term in the right side involves the point process for the ideal gas, while permutation jumps are interacting. It can be easily calculated numerically since the set of permutations is discrete. To know the corresponding linear behaviour would already provide some information. The first term involves the positions of particles, and it may be difficult to generate a typical realisation of the points. In any case, the random permutation approach with Eq. (6.10) 
is much easier than path-integral Monte-Carlo simulations, and it should yield the same result to lowest order.

\section{A SIMPLE MODEL OF SPATIAL RANDOM PERMUTATIONS WITH INTERACTIONS}

In the preceding section we have discussed a two-body interaction that is exactly related to the quantum Bose gas. Rigorous results seem difficult to get, though. In this section we simplify the interaction so as to retain only the largest contribution. Our approximation is not exact, but the model may be of interest as an effective model, and it is exactly solvable.

7.1. Approximation and definition of the model. It helps to think of particles as describing Brownian motions, and to interact whenever they cross each other's paths. Clearly, particles that belong to the same 2-cycle interact a lot. Our approximation consists in retaining only these interactions, and in neglecting the rest. Thus we consider the Hamiltonian

$$
\tilde{H}(\boldsymbol{x}, \pi)=\frac{1}{4 \beta} \sum_{i=1}^{N}\left|x_{i}-x_{\pi(i)}\right|^{2}+\sum_{\substack{1 \leqslant i<j \leqslant N \\ \pi(i)=j, \pi(j)=i}} V\left(x_{i}, x_{j}, x_{j}, x_{i}\right) .
$$

The potential $V(\cdot)$ is the exact jump interaction given in 6.10 . To lowest order in the scattering length, we find that [4]

$$
V(x, y, y, x)=\frac{2 a}{|x-y|}+O\left(a^{2}\right)
$$

If $\theta$ is a random variable that depends only on permutations, its expectation is given by

$$
E_{\Lambda, N}(\theta)=\frac{1}{Z(\Lambda, N) N !} \sum_{\pi \in \mathcal{S}_{N}} \theta(\pi) \int_{\Lambda^{N}} \mathrm{~d} \boldsymbol{x} \mathrm{e}^{-\tilde{H}(\boldsymbol{x}, \pi)} .
$$

This allows to simplify the model 7.1 further, without additional approximation. Namely, we introduce the simpler Hamiltonian

$$
H^{(\alpha)}(\boldsymbol{x}, \pi)=\frac{1}{4 \beta} \sum_{i=1}^{N}\left|x_{i}-x_{\pi(i)}\right|^{2}+\alpha N_{2}(\pi),
$$

with $N_{2}(\pi)$ denoting the number of 2-cycles in the permutation $\pi$. Expectations with $\tilde{H}$ and $H^{(\alpha)}$ are identical provided

$$
\int_{\Lambda^{N}} \mathrm{~d} \boldsymbol{x} \mathrm{e}^{-\tilde{H}(\boldsymbol{x}, \pi)}=\int_{\Lambda^{N}} \mathrm{~d} \boldsymbol{x} \mathrm{e}^{-H^{(\alpha)}(\boldsymbol{x}, \pi)}
$$

for any fixed permutation $\pi$. Both sides factorise according to the cycles of $\pi$. The contribution of cycles of length $1,3,4, \ldots$ is identical. We then obtain an equation for cycles of length 2 , namely

$$
\int_{\Lambda^{2}} \mathrm{~d} x_{1} \mathrm{~d} x_{2} \mathrm{e}^{-\frac{1}{2 \beta}\left|x_{1}-x_{2}\right|^{2}-V\left(x_{1}, x_{2}, x_{2}, x_{1}\right)}=\int_{\Lambda^{2}} \mathrm{~d} x_{1} \mathrm{~d} x_{2} \mathrm{e}^{-\frac{1}{2 \beta}\left|x_{1}-x_{2}\right|^{2}-\alpha} .
$$

Using (7.2), a few computations give [4]

$$
\alpha=\left(\frac{8}{\pi \beta}\right)^{1 / 2} a+O\left(a^{2}\right) .
$$


We emphasise that the approximation consists in retaining only interactions within 2cycles. Computations are exact afterwards, at least to lowest order in the scattering length of the interaction potential $U$ between the quantum particles.

7.2. Pressure and critical density. We now generalise a bit the model above by considering more general one-body terms, as we have done throughout this article. Let

$$
H^{(\alpha)}(\boldsymbol{x}, \pi)=\sum_{i=1}^{N} \xi_{\Lambda}\left(x_{i}-x_{\pi(i)}\right)+\alpha N_{2}(\pi)
$$

with $\xi_{\Lambda}$ as in Section 5 , and $\alpha$ is a positive parameter.

The pressure of this simple model can be computed exactly. One gets the critical density by analogy with the ideal gas. We state below a result about infinite cycles for a larger density, see Theorem 7.2. It remains an open problem to show that infinite cycles are present all the way to the critical density.

For given $\alpha$, the pressure depends on the chemical potential $\mu$ and is given by

$$
p^{(\alpha)}(\mu)=\lim _{V \rightarrow \infty} \frac{1}{V} \log Z(\Lambda, \mu)
$$

with $Z(\Lambda, \mu)$ the "grand-canonical partition function". We can define it directly in the Fourier space, by

$$
Z(\Lambda, \mu)=\sum_{N \geqslant 0} \frac{\mathrm{e}^{\mu N}}{N !} \sum_{k_{1}, \ldots, k_{N} \in \Lambda^{*}} \sum_{\pi \in \mathcal{S}_{N}} \mathrm{e}^{-\alpha N_{2}(\pi)} \prod_{i=1}^{N} \mathrm{e}^{-\varepsilon\left(k_{i}\right)} \delta_{k_{i}, k_{\pi(i)}} .
$$

We need the pressure of the ideal gas

$$
p^{(0)}(\mu)=-\int_{\mathbb{R}^{d}} \log \left(1-\mathrm{e}^{-(\varepsilon(k)-\mu)}\right) \mathrm{d} k .
$$

Theorem 7.1. For $\mu<0$, the limit (7.9) exists, and

$$
p^{(\alpha)}(\mu)=p^{(0)}(\mu)-\frac{1}{2} \mathrm{e}^{2 \mu}\left[1-\mathrm{e}^{-\alpha}\right] \int_{\mathbb{R}^{d}} \mathrm{e}^{-2 \varepsilon(k)} \mathrm{d} k .
$$

Notice that the model is defined only for $\mu<0$, like the ideal gas. Its derivative at $\mu=0-$ gives the critical density, and we find

$$
\rho_{\mathrm{c}}^{(\alpha)}=\left.\frac{\partial p^{(\alpha)}}{\partial \mu}\right|_{\mu=0-}=\rho_{\mathrm{c}}^{(0)}-\left[1-\mathrm{e}^{-\alpha}\right] \int \mathrm{e}^{-2 \varepsilon(k)} \mathrm{d} k .
$$

The first term of the right side is equal to the critical density of the ideal gas, Eq. (5.8). The second term is the correction due to our simple interaction.

The physically relevant situation is $d=3, \xi(x)=|x|^{2} / 4 \beta$, and $\alpha$ in (7.7). We find that, to lowest order in $a$,

$$
\frac{T_{\mathrm{c}}^{(a)}-T_{\mathrm{c}}^{(0)}}{T_{\mathrm{c}}^{(0)}} \approx \tilde{c} \rho^{1 / 3} a,
$$

with $\tilde{c}=0.37$. The details of the computations can be found in [4]. This formula can be compared with (6.11). Our constant has the correct sign, and is about a quarter of the expected constant. This suggests that the simplified model accounts for some of the effects of interactions on the critical temperature for Bose-Einstein condensation. Besides, it allows for simple but illuminating heuristics: Interactions discourage small cycles; then all other cycles are favoured, including infinite cycles. As a consequence, repulsive interactions increase the critical temperature (or decrease the critical density). 
Proof of Theorem 7.1. From 7.10, we have

$$
Z(\Lambda, \mu)=\sum_{\left(n_{k}\right)_{k \in \Lambda^{*}}} \prod_{k \in \Lambda^{*}}\left[\mathrm{e}^{-(\varepsilon(k)-\mu) n_{k}} \sum_{\pi_{k} \in \mathcal{S}_{n_{k}}} \frac{1}{n_{k} !} \mathrm{e}^{-\alpha N_{2}\left(\pi_{k}\right)}\right] .
$$

We decomposed the permutation $\pi$ into permutations $\left(\pi_{k}\right)$ for each Fourier mode, and we also used

$$
N_{2}(\pi)=\sum_{k \in \Lambda^{*}} N_{2}\left(\pi_{k}\right)
$$

Notice that the chemical potential needs to be strictly negative, as in the ideal gas. We get

$$
p^{(\alpha)}(\mu)=\lim _{V \rightarrow \infty} \frac{1}{V} \sum_{k \in \Lambda^{*}} \log \left[\sum_{n \geqslant 0} \mathrm{e}^{-(\varepsilon(k)-\mu) n} \sum_{\pi \in \mathcal{S}_{n}} \frac{1}{n !} \mathrm{e}^{-\alpha N_{2}(\pi)}\right] .
$$

Let us compute the bracket above. For given $\pi \in \mathcal{S}_{n}$, let $r_{j}$ denote the number of cycles of length $j$. Then $\sum_{j} j r_{j}=n$, and the number of permutations for given $\left(r_{j}\right)$ is equal to

$$
n ! / \prod_{j \geqslant 1} j^{r_{j}} r_{j} !
$$

The bracket in 7.16 is then equal to

$$
\begin{aligned}
& \sum_{n \geqslant 0} \frac{1}{n !} \sum_{\substack{r_{1}, r_{2}, \ldots \\
\sum_{j} j r_{j}=n}} \frac{n !}{\prod_{j \geqslant 1} j^{r_{j} r_{j} !}} \mathrm{e}^{-(\varepsilon(k)-\mu) \sum_{j} j r_{j}} \mathrm{e}^{-\alpha r_{2}} \\
= & \sum_{r_{1}, r_{3}, r_{4}, \ldots \geqslant 0} \prod_{j=1,3,4, \ldots} \frac{1}{r_{j} !}\left[\frac{1}{j} \mathrm{e}^{-j(\varepsilon(k)-\mu)}\right]^{r_{j}} \sum_{r_{2} \geqslant 0} \frac{1}{r_{2} !}\left[\frac{1}{2} \mathrm{e}^{-2(\varepsilon(k)-\mu)-\alpha}\right]^{r_{2}} \\
= & \exp \left\{\sum_{j=1,3,4, \ldots} \frac{1}{j} \mathrm{e}^{-j(\varepsilon(k)-\mu)}+\frac{1}{2} \mathrm{e}^{-2(\varepsilon(k)-\mu)-\alpha}\right\} \\
= & \exp \left\{-\log \left(1-\mathrm{e}^{-(\varepsilon(k)-\mu)}\right)-\frac{1}{2} \mathrm{e}^{-2(\varepsilon(k)-\mu)}\left[1-\mathrm{e}^{-\alpha}\right]\right\} .
\end{aligned}
$$

We can insert this into (7.16). In the limit $V \rightarrow \infty$ the expression converges to a Riemann integral.

7.3. Occurrence of infinite cycles. Given $\alpha \in[0, \infty]$, the expectation of a random variable on permutations is given by

$$
E_{\Lambda, N}(\theta)=\frac{1}{Z(\Lambda, N) N !} \sum_{\pi \in \mathcal{S}_{N}} \theta(\pi) \mathrm{e}^{-\alpha N_{2}(\pi)} \int_{\Lambda^{N}} \mathrm{~d} x_{1} \ldots \mathrm{d} x_{N} \prod_{i=1}^{N} \mathrm{e}^{-\xi_{\Lambda}\left(x_{i}-x_{\pi(i)}\right)} .
$$

The normalisation $Z(\Lambda, N)$ depends on $\alpha$, although the notation does not make it explicit. We expect that the claims of Theorem 5.1 extend to $\alpha \neq 0$, with the critical density given by $(7.12)$ instead of $(5.8)$. But we only state and prove a weaker claim.

Theorem 7.2. For all $0<b<1$,

$$
\lim _{V \rightarrow \infty} E_{\Lambda, \rho V}\left(\varrho_{V^{b}, \rho V}\right) \geqslant \rho-\frac{4}{\left(1+\mathrm{e}^{-\alpha}\right)^{2}} \rho_{\mathrm{c}}^{(0)} .
$$


Of course, the theorem is useful only if the right side is strictly positive. The proof is similar to Theorem 5.1, but there is one important difference. We cannot invoke a set of typical occupation numbers, such as $A_{\eta}$ in Eq. (5.24). We prove below (Proposition 7.6) that the zero Fourier mode is macroscopically occupied if the density is large enough. But we need it to be strictly bigger than $\rho_{\mathrm{c}}^{(0)}$, itself bigger than $\rho_{\mathrm{c}}^{(\alpha)}$.

Let us define

$$
h_{n}(\alpha)=\frac{1}{n !} \sum_{\pi \in \mathcal{S}_{n}} \mathrm{e}^{-\alpha N_{2}(\pi)} .
$$

Notice that $h_{n}(0)=1$. We introduce a probability on $\left(\Lambda^{*}\right)^{N} \times \mathcal{S}_{N}$ that generalises Eq. (5.14):

$$
P_{\Lambda, N}(\boldsymbol{k} \times \pi)=\frac{1}{Z^{\prime}(\Lambda, N) N !} \mathrm{e}^{-\alpha N_{2}(\pi)} \mathrm{e}^{-\sum_{i=1}^{N} \varepsilon\left(k_{i}\right)}
$$

if $k_{i}=k_{\pi(i)}$ for all $i$, it is zero otherwise. Summing over permutations, and over vectors $\boldsymbol{k}$ that are compatibles with $\boldsymbol{n}$, we get

$$
P_{\Lambda, N}(\boldsymbol{n})=\frac{1}{Z^{\prime}(\Lambda, N)} \prod_{k \in \Lambda^{*}} \mathrm{e}^{-\varepsilon(k)} h_{n_{k}}(\alpha) .
$$

We generalise Lemma 5.4 .

Lemma 7.3. We have $Z(\Lambda, N)=C^{N} Z^{\prime}(\Lambda, N)$ with $C=\int \mathrm{e}^{-\xi}$; and for all $\theta \in \mathcal{R}_{N}$,

$$
E_{\Lambda, N}(\theta)=\sum_{\boldsymbol{n} \in \mathcal{N}_{\Lambda, N}} P_{\Lambda, N}(\boldsymbol{n}) \sum_{\pi \in \mathcal{S}_{N}} \theta(\pi) P_{\Lambda, N}(\pi \mid \boldsymbol{k})
$$

with $\boldsymbol{k}$ any $N$-tuple such that $\boldsymbol{n}(\boldsymbol{k})=\boldsymbol{n}$.

Proof. We use Corollary 5.3 to rewrite the expectation $E_{\Lambda, N}$ in the Fourier space:

$$
\begin{aligned}
E_{\Lambda, N}(\theta) & =\frac{1}{Z(\Lambda, N) N !} \sum_{\pi \in \mathcal{S}_{N}} \theta(\pi) \mathrm{e}^{-\alpha N_{2}(\pi)} \sum_{k_{1}, \ldots, k_{N} \in \Lambda^{*}} \prod_{i=1}^{N} \delta_{k_{i}, k_{\pi(i)}} C \mathrm{e}^{-\varepsilon\left(k_{i}\right)} \\
& =\sum_{\pi \in \mathcal{S}_{N}} \theta(\pi) \sum_{\boldsymbol{k} \in\left(\Lambda^{*}\right)^{N}} P_{\Lambda, N}(\boldsymbol{k} \times \pi) \\
& =\sum_{\boldsymbol{k} \in\left(\Lambda^{*}\right)^{N}} P_{\Lambda, N}(\boldsymbol{k}) \sum_{\pi \in \mathcal{S}_{N}} \theta(\pi) P_{\Lambda, N}(\pi \mid \boldsymbol{k}) .
\end{aligned}
$$

One can sum first over $\boldsymbol{n}$ and then over compatible $\boldsymbol{k}$ 's.

Next we gather some information on the functions $h_{n}(\alpha)$ defined in (7.18).

Lemma 7.4. For $\alpha \in[0, \infty]$, let $\delta=\frac{1}{2}\left(1-\mathrm{e}^{-\alpha}\right) \in\left[0, \frac{1}{2}\right]$.

(a) $h_{n}(\alpha)=\sum_{j=0}^{\left\lfloor\frac{n}{2}\right\rfloor} \frac{1}{j !}(-\delta)^{j}$.

(b) $1-\delta \leqslant h_{n}(\alpha) \leqslant 1$.

(c) $\mathrm{e}^{-\delta}-\delta^{n / 2} /\left\lfloor\frac{n}{2}\right\rfloor ! \leqslant h_{n}(\alpha) \leqslant \mathrm{e}^{-\delta}+\delta^{n / 2} /\left\lfloor\frac{n}{2}\right\rfloor$ !.

Proof. Isolating the contribution of the cycle that contains 1, we get the following recursive relation; for $n \geqslant 2$,

$$
h_{n}(\alpha)=\frac{1}{n} \sum_{j=0}^{n-1} h_{j}(\alpha)-\frac{1}{n}\left(1-\mathrm{e}^{-\alpha}\right) h_{n-2}(\alpha) .
$$


We also have $h_{0}(\alpha)=h_{1}(\alpha)=1$. Now the formula in (a) can be proved by induction. (b) is a consequence of the alternating series in (a). Notice that $h_{2}(\alpha)=h_{3}(\alpha)=1-\delta$. (c) follows from the expression

$$
h_{n}(\alpha)=\mathrm{e}^{-\delta}-\sum_{j \geqslant\left\lfloor\frac{n}{2}\right\rfloor+1} \frac{(-\delta)^{j}}{j !} .
$$

Recall that alternating series of decreasing terms are bounded by their first term.

Let us define $N_{a, n}(\pi)=\sum_{i=1}^{n} \chi_{[a, n]}\left(\ell_{i}(\pi)\right)$. Note that $N_{a, n}(\pi)=V \varrho_{a, n}(\pi)$.

Lemma 7.5. Suppose $a>2$, and let $\delta$ as in Lemma 7.4. For all $m \geqslant 0$,

$$
\sum_{\pi \in \mathcal{S}_{n}} N_{a, n}(\pi) \frac{\mathrm{e}^{-\alpha N_{2}(\pi)}}{h_{n}(\alpha) n !} \geqslant(n-a-2 m)\left(1-\delta^{m}\right) .
$$

Proof. When summing over permutations, all indexes $i$ in the definition of $N_{a, n}(\pi)$ are equivalent, so that

$$
\sum_{\pi \in \mathcal{S}_{n}} N_{a, n}(\pi) \frac{\mathrm{e}^{-\alpha N_{2}(\pi)}}{h_{n}(\alpha) n !}=n \sum_{\pi \in \mathcal{S}_{n}} \chi_{[a, n]}\left(\ell_{1}(\pi)\right) \frac{\mathrm{e}^{-\alpha N_{2}(\pi)}}{h_{n}(\alpha) n !} .
$$

Summing over the lengths of the cycle that contains 1, we get

$$
\begin{aligned}
\sum_{\pi \in \mathcal{S}_{n}} N_{a, n}(\pi) \frac{\mathrm{e}^{-\alpha N_{2}(\pi)}}{h_{n}(\alpha) n !} & =\sum_{j=a}^{n} n(n-1) \ldots(n-j+1) \sum_{\pi \in \mathcal{S}_{n-j}} \frac{\mathrm{e}^{-\alpha N_{2}(\pi)}}{h_{n}(\alpha) n !} \\
& =\sum_{j=a}^{n} \sum_{\pi \in \mathcal{S}_{n-j}} \frac{\mathrm{e}^{-\alpha N_{2}(\pi)}}{h_{n-j}(\alpha)(n-j) !} \frac{h_{n-j}(\alpha)}{h_{n}(\alpha)} .
\end{aligned}
$$

We get a lower bound by summing up to $n-2 m$. By Lemma 7.4 (c), we have for $2<j<n-2 m$

$$
\frac{h_{n-j}(\alpha)}{h_{n}(\alpha)} \geqslant \frac{\mathrm{e}^{-\delta}-\delta^{m} / m !}{\mathrm{e}^{-\delta}+\delta^{n / 2} /\left\lfloor\frac{n}{2}\right\rfloor !} \geqslant 1-\delta^{m} .
$$

The last result that is needed for the proof of Theorem 7.2 is that our interacting model displays Bose-Einstein condensation, in the sense that the zero Fourier mode is macroscopically occupied.

Proposition 7.6. The expectation for the occupation of the zero Fourier mode is bounded below by

$$
\lim _{V \rightarrow \infty} E_{\Lambda, N}\left(\frac{n_{0}}{V}\right) \geqslant \rho-\frac{4}{\left(1+\mathrm{e}^{-\alpha}\right)^{2}} \rho_{\mathrm{c}}^{(0)} .
$$

Proof. We proceed as in Appendix B of [27]. We have

$$
E_{\Lambda, N}\left(\frac{n_{0}}{V}\right)=\rho-\frac{1}{V} \sum_{k \in \Lambda^{*} \backslash\{0\}} E_{\Lambda, N}\left(n_{k}\right),
$$


and

$$
\begin{aligned}
E_{\Lambda, N}\left(n_{k}\right) & =\sum_{j \geqslant 1} P_{\Lambda, N}\left(n_{k} \geqslant j\right) \\
& =\sum_{j \geqslant 1} \sum_{n \in \mathcal{N}_{\Lambda, N-j}} \mathrm{e}^{-\varepsilon(k) j} \frac{\prod_{k^{\prime} \in \Lambda^{*}} \mathrm{e}^{-\varepsilon\left(k^{\prime}\right) n_{k^{\prime}} h_{n_{k^{\prime}}}(\alpha)}}{Z^{\prime}(\Lambda, N)} \frac{h_{n_{k}+j}(\alpha)}{h_{n_{k}}(\alpha)} .
\end{aligned}
$$

The latter ratio is smaller than $(1-\delta)^{-1}$ by Lemma 7.4 (b). By restricting occupation numbers to $n_{0} \geqslant j$, we also have

$$
\begin{aligned}
Z^{\prime}(\Lambda, N) & \geqslant \sum_{\boldsymbol{n} \in \mathcal{N}_{\Lambda, N-j}} \prod_{k \in \Lambda^{*}} \mathrm{e}^{-\varepsilon(k) n_{k}} h_{n_{k}}(\alpha) \frac{h_{n_{0}+j}(\alpha)}{h_{n_{0}}(\alpha)} \\
& \geqslant Z^{\prime}(\Lambda, N-j)(1-\delta) .
\end{aligned}
$$

Then

$$
E_{\Lambda, N}\left(n_{k}\right) \leqslant(1-\delta)^{-2} \sum_{j \geqslant 1} \mathrm{e}^{-\varepsilon(k) j}=(1-\delta)^{-2} \frac{1}{\mathrm{e}^{\varepsilon(k)}-1} .
$$

It follows that

$$
E_{\Lambda, N}\left(\frac{n_{0}}{V}\right) \geqslant \rho-(1-\delta)^{-2} \frac{1}{V} \sum_{k \in \Lambda^{*} \backslash\{0\}} \frac{1}{\mathrm{e}^{\varepsilon(k)}-1} .
$$

We get the proposition by letting $V \rightarrow \infty$.

Proof of Theorem 7.2. From Lemma 7.3, we have

$$
E_{\Lambda, N}\left(\varrho_{m, n}\right)=\sum_{\boldsymbol{n} \in \mathcal{N}_{\Lambda, N}} P_{\Lambda, N}(\boldsymbol{n}) \sum_{\substack{\pi \in \mathcal{S}_{N} \\ k_{i}=k_{\pi(i)} \forall i}} \boldsymbol{\varrho}_{m, n}(\pi) \frac{\mathrm{e}^{-\alpha N_{2}(\pi)}}{\prod_{k \in \Lambda^{*}} h_{n_{k}}(\alpha) n_{k} !} .
$$

Compatible permutations factorise according to Fourier modes, i.e. $\pi=\left(\pi_{k}\right)$ with $\pi_{k} \in$ $\mathcal{S}_{n_{k}}$. Also, $N_{2}(\pi)=\sum_{k} N_{2}\left(\pi_{k}\right)$. Then

$$
\begin{aligned}
E_{\Lambda, N}\left(\boldsymbol{\varrho}_{m, n}\right) & =\sum_{\boldsymbol{n} \in \mathcal{N}_{\Lambda, N}} P_{\Lambda, N}(\boldsymbol{n})\left(\prod_{k \in \Lambda^{*}} \sum_{\pi_{k} \in \mathcal{S}_{n_{k}}} \frac{\mathrm{e}^{-\alpha N_{2}\left(\pi_{k}\right)}}{h_{n_{k}}(\alpha) n_{k} !}\right) \varrho_{m, n}\left(\left(\pi_{k}\right)\right) \\
& =\sum_{\boldsymbol{n} \in \mathcal{N}_{\Lambda, N}} P_{\Lambda, N}(\boldsymbol{n}) \sum_{k \in \Lambda^{*}} \sum_{\pi_{k} \in \mathcal{S}_{n_{k}}} \boldsymbol{\varrho}_{m, n}\left(\pi_{k}\right) \frac{\mathrm{e}^{-\alpha N_{2}\left(\pi_{k}\right)}}{h_{n_{k}}(\alpha) n_{k} !}
\end{aligned}
$$

We keep only the term $k=0$. Using Lemma 7.5, we obtain the lower bound

$$
\begin{aligned}
E_{\Lambda, N}\left(\boldsymbol{\varrho}_{V^{b}, N}\right) & \geqslant \sum_{\boldsymbol{n} \in \mathcal{N}_{\Lambda, N}} P_{\Lambda, N}(\boldsymbol{n}) \frac{n_{0}-3 V^{b}}{V}\left(1-\delta^{V^{b}}\right) \\
& \geqslant E_{\Lambda, N}\left(\frac{n_{0}}{V}\right)-4 V^{b-1} .
\end{aligned}
$$

The claim follows from Proposition 7.6. 


\section{Appendix A. Macroscopic occupation of the Zero Fourier mode.}

In this appendix we investigate the random variable $\boldsymbol{n} \mapsto n_{0}$ under the measure (5.16) in the thermodynamic limit $V \rightarrow \infty, N=\rho V$, for all density parameters $\rho$. We want to show that $n_{0} / V$ approaches a limit for each density $\rho$. We will actually show much more, by giving the limiting moment generating function of $n_{0} / V$. We partly follow Buffet and Pulé [6], who considered the ideal Bose gas in arbitrary domains.

Theorem A.1. Let $\rho_{0}=\max \left(0, \rho-\rho_{\mathrm{c}}\right)$, with $\rho_{\mathrm{c}}$ the critical density defined in (5.8). Then

$$
\lim _{V \rightarrow \infty} E_{\Lambda, \rho V}\left(\mathrm{e}^{\lambda n_{0} / V}\right)=\mathrm{e}^{\lambda \rho_{0}}
$$

for all $\lambda \geqslant 0$.

Our first proof applies only when $\rho_{c}$ is finite. We give below an argument that completes the proof.

Proof when $\rho_{\mathrm{c}}<\infty$. It is shown in [27], Appendix B, that for all $k \in \Lambda^{*}$, we have

$$
P_{\Lambda, N}\left(n_{k} \geqslant j\right)=\mathrm{e}^{-\varepsilon(k) j} \frac{Z^{\prime}(\Lambda, N-j)}{Z^{\prime}(\Lambda, N)} .
$$

Since $P\left(n_{k}=j\right)=P\left(n_{k} \geqslant j\right)-P\left(n_{k} \geqslant j+1\right)$, we find for $b>0$

$$
\begin{aligned}
E_{\Lambda, N}\left(\mathrm{e}^{\nu n_{0}}\right) & =\frac{1}{Z^{\prime}(\Lambda, N)} \sum_{j=0}^{N} \mathrm{e}^{\nu j}\left(Z^{\prime}(\Lambda, N-j)-Z^{\prime}(\Lambda, N-j-1)\right) \\
& =\frac{\mathrm{e}^{\nu N}}{Z^{\prime}(\Lambda, N)} \sum_{j=0}^{N} \mathrm{e}^{-\nu j}\left(Z^{\prime}(\Lambda, j)-Z^{\prime}(\Lambda, j-1)\right) .
\end{aligned}
$$

Here, we used the convention $Z(\Lambda,-1):=0$ and the fact that $P_{\Lambda, N}\left(n_{k} \geqslant N+1\right)=0$. Putting in $N=\lfloor\rho V\rfloor$ and setting $\nu=\lambda / V$ we obtain

$$
E_{\Lambda, \rho V}\left(\mathrm{e}^{\lambda n_{0} / V}\right)=\frac{\mathrm{e}^{\lambda \rho}}{Z^{\prime}(\Lambda, \rho V)} \sum_{j=0}^{\rho \Lambda} \mathrm{e}^{-\frac{\lambda}{V} j}\left(Z^{\prime}(\Lambda, j)-Z^{\prime}(\Lambda, j-1)\right) .
$$

Above, we wrote $\rho V$ instead of $\lfloor\rho V\rfloor$ and we will continue to do so, to simplify notation. Now comes the clever insight of Buffet and Pulé [6]: In $(\mathrm{A} .3)$, both $Z^{\prime}(\Lambda, \rho V)$ and the sum over $j$ can be written as integrals with respect to a purely atomic, $V$-dependent measure $\mu_{\Lambda}$ on $\mathbb{R}^{+}$; since the functions that are being integrated will not depend on $V$, we only need to study the limit of $\mu_{\Lambda}$. The measure $\mu_{\Lambda}$ is given by

$$
\mu_{\Lambda}:=C_{\Lambda} \sum_{j=0}^{\infty}\left(Z^{\prime}(\Lambda, j)-Z^{\prime}(\Lambda, j-1)\right) \delta_{j / V}
$$

on $\mathbb{R}^{+} . \delta_{x}$ denotes the Dirac measure at $x$, and the constant $C_{\Lambda}$ will be fixed later in order to obtain a limit measure. From A.3 it is now immediate that

$$
E_{\Lambda, \rho V}\left(\mathrm{e}^{\lambda n_{0} / V}\right)=\mathrm{e}^{\lambda \rho} \frac{\int 1_{[0, \rho]}(x) \mathrm{e}^{-\lambda x} \mu_{\Lambda}(\mathrm{d} x)}{\int 1_{[0, \rho]}(x) \mu_{\Lambda}(\mathrm{d} x)} .
$$


What makes the idea work is that we can actually calculate the Laplace transform of $\mu_{\Lambda}$ and take the limit. We have

$$
\begin{aligned}
\int_{0}^{\infty} \mathrm{e}^{-\lambda x} \mu_{\Lambda}(\mathrm{d} x) & =C_{\Lambda} \sum_{j=0}^{\infty} \mathrm{e}^{-\frac{\lambda j}{V}}\left(Z^{\prime}(\Lambda, j)-Z^{\prime}(\Lambda, j-1)\right)= \\
& =C_{\Lambda}\left(1-\mathrm{e}^{-\frac{\lambda}{V}}\right) \sum_{j=0}^{\infty} \mathrm{e}^{-\frac{\lambda j}{V}} Z^{\prime}(\Lambda, j)= \\
& =C_{\Lambda}\left(1-\mathrm{e}^{-\frac{\lambda}{V}}\right) \exp \left(-\sum_{k \in \Lambda^{*}} \log \left(1-\mathrm{e}^{-\frac{\lambda}{V}-\varepsilon(k)}\right)\right)= \\
& =C_{\Lambda} \exp \left(-\sum_{k \in \Lambda^{*} \backslash\{0\}} \log \left(1-\mathrm{e}^{-\frac{\lambda}{V}-\varepsilon(k)}\right)\right)
\end{aligned}
$$

The second equality above is just an index shift, the third is the well-known formula for the pressure of the ideal Bose gas, Eq. (7.11), and the last line follows from $\varepsilon(0)=0$. The sum in the exponent of the last line is actually quite manageable. By the fundamental theorem of calculus we have for each $k \neq 0$

$$
\log \left(1-\mathrm{e}^{-\frac{\lambda}{V}-\varepsilon(k)}\right)=\frac{1}{V} \int_{0}^{\lambda} \frac{1}{\mathrm{e}^{y / V+\varepsilon(k)}-1} \mathrm{~d} y+\log \left(1-\mathrm{e}^{-\varepsilon(k)}\right),
$$

and summation over $k$ gives

$$
\sum_{k \in \Lambda^{*} \backslash\{0\}} \log \left(1-\mathrm{e}^{-\frac{\lambda}{V}-\varepsilon(k)}\right)=\sum_{k \in \Lambda^{*} \backslash\{0\}} \log \left(1-\mathrm{e}^{-\varepsilon(k)}\right)+\lambda \rho_{\mathrm{c}, \Lambda}
$$

with

$$
\rho_{\mathrm{c}, \Lambda}=\frac{1}{\lambda} \int_{0}^{\lambda} \frac{1}{V} \sum_{k \in \Lambda^{*} \backslash\{0\}} \frac{1}{\mathrm{e}^{y / V+\varepsilon(k)}-1} \mathrm{~d} y
$$

The first term in A.5 diverges as $V \rightarrow \infty$ and defines $C_{\Lambda}$. The second term converges to the critical density $\rho_{\mathrm{c}}$ : the integrand is decreasing as a function of $y$ and converges to $\rho_{\mathrm{c}}$ as a Riemann sum for each fixed $y$, since

$$
\mathrm{e}^{-y / V} \frac{1}{V} \sum_{k \in \Lambda^{*} \backslash\{0\}} \frac{1}{\mathrm{e}^{\varepsilon(k)}-1} \leqslant \frac{1}{V} \sum_{k \in \Lambda^{*} \backslash\{0\}} \frac{1}{\mathrm{e}^{y / V+\varepsilon(k)}-1} \leqslant \frac{1}{V} \sum_{k \in \Lambda^{*} \backslash\{0\}} \frac{1}{\mathrm{e}^{\varepsilon(k)}-1} .
$$

Dominated convergence in $y$ now proves convergence to $\rho_{\mathrm{c}}$. We have thus shown that for all $\lambda>0$

$$
\lim _{V \rightarrow \infty} \int_{0}^{\infty} e^{-\lambda x} \mu_{\Lambda}(\mathrm{d} x)=\mathrm{e}^{-\lambda \rho_{\mathrm{c}}}
$$

and thus by the general theory of Laplace transforms $\mu_{\Lambda} \rightarrow \delta_{\rho_{\mathrm{c}}}$ weakly. When used in A.4, this shows the claim for $\rho>\rho_{\mathrm{c}}$. For $\rho<\rho_{\mathrm{c}}$, both denominator and numerator go to zero, and we need a different argument: we note that by what we have just proved

$$
\lim _{\rho \searrow \rho_{\mathrm{c}}} \lim _{V \rightarrow \infty} E_{\Lambda, \rho V}\left(\mathrm{e}^{\lambda n_{0} / V}\right)=1 .
$$

Since the expectation above can never be less than one, all we need to show is monotonicity in $\rho$, i.e.

$$
P_{\Lambda, N+1}\left(n_{0} \geqslant j\right) \geqslant P_{\Lambda, N}\left(n_{0} \geqslant j\right) .
$$


For this we use (A.1) and we obtain

$$
P_{\Lambda, N+1}\left(n_{k} \geqslant j\right)=P_{\Lambda, N}\left(n_{k} \geqslant j\right) \frac{P_{\Lambda, N+1}\left(n_{k} \geqslant 1\right)}{P_{\Lambda, N-j+1}\left(n_{k} \geqslant 1\right)},
$$

so it will be enough to show A.6 for $j=1$. By A.1 this means we have to show

$$
Z^{\prime}(\Lambda, N)^{2} \geqslant Z^{\prime}(\Lambda, N-1) Z^{\prime}(\Lambda, N+1)
$$

Davies [7] showed that the finite volume free energy is convex, which proves the inequality above.

Proof of Theorem A.1 when $\rho_{\mathrm{c}}=\infty$. We get from Eq. A.2, after some rearrangements of the terms

$$
\left|E_{\Lambda, N}\left(\mathrm{e}^{\lambda n_{0} / V}\right)-\mathrm{e}^{-\lambda / V}\right|=\left(1-\mathrm{e}^{-\lambda / V}\right) \mathrm{e}^{\lambda \rho} \sum_{i=0}^{N} \mathrm{e}^{-\lambda i / V} \mathrm{e}^{-V\left[q_{\Lambda}(i / V)-q_{\Lambda}(N / V)\right]} .
$$

$q_{\Lambda}(\rho)$ is convex and its limit $q(\rho)$ is strictly decreasing for $\rho<\rho_{\mathrm{c}}$ (and $\rho_{\mathrm{c}}=\infty$ here). Beside, we have $q_{\Lambda}(i / V)-q_{\Lambda}(N / V) \geqslant b>0$, for all $0 \leqslant i \leqslant N / 2$, uniformly in $V$ and $N=\rho V$. The right side of A.7) is then less than

$$
\left(1-\mathrm{e}^{-\lambda / V}\right) \mathrm{e}^{\lambda \rho}\left[\sum_{i=0}^{N / 2} \mathrm{e}^{-V b}+\sum_{i=N / 2}^{N} \mathrm{e}^{-\lambda i / V}\right] .
$$

This clearly vanishes in the limit $V \rightarrow \infty$.

Recall the definition of the typical set of occupation numbers $A_{\eta}$ in (5.24).

Proposition A.2. For any density $\rho$, and any $\eta>0$,

$$
\lim _{V \rightarrow \infty} P_{\Lambda, \rho V}\left(A_{\eta}\right)=1 \text {. }
$$

Proof. Let us introduce the following sets of unlikely occupation numbers:

$$
\begin{aligned}
& A^{(1)}=\left\{\left(n_{k}\right):\left|n_{0} / V-\rho_{0}\right|>\eta\right\}, \\
& A^{(2)}=\left\{\left(n_{k}\right): \sum_{0<|k|<V^{-\eta}} n_{k} \geqslant \eta V\right\}, \\
& A^{(3)}=\left\{\left(n_{k}\right): n_{k} \geqslant V^{3 \eta} \text { for some }|k| \geqslant V^{-\eta}\right\} .
\end{aligned}
$$

Then

$$
A_{\eta}^{\mathrm{c}}=A^{(1)} \cup A^{(2)} \cup A^{(3)} .
$$

It follows from Theorem A.1 that $P_{\Lambda, \rho V}\left(A^{(1)}\right)$ vanishes in the limit $V \rightarrow \infty$. Equation A.1) can be written using free energies as

$$
P_{\Lambda, N}\left(n_{k} \geqslant i\right)=\mathrm{e}^{-\varepsilon(k) i} \mathrm{e}^{-V\left(q_{\Lambda}\left(\frac{N-i}{V}\right)-q_{\Lambda}\left(\frac{N}{V}\right)\right)} .
$$

We have already mentioned that $q_{\Lambda}$ is convex, and the limit $q$ is decreasing. Then

$$
q_{\Lambda}(\rho-\epsilon)-q_{\Lambda}(\rho) \geqslant \nu
$$

with $\nu \geqslant 0$. In addition, $\nu>0$ below the critical density, or if the critical density is infinite. Since

$$
E_{\Lambda, N}\left(n_{k}\right)=\sum_{i \geqslant 0} P_{\Lambda, N}\left(n_{k} \geqslant i\right)
$$


we get a bound for the expectation of occupation numbers for $k \neq 0$, namely

$$
E_{\Lambda, N}\left(n_{k}\right) \leqslant \frac{1}{\mathrm{e}^{\varepsilon(k)+\nu}-1} .
$$

From Markov's inequality and the bound above, we get

$$
\begin{aligned}
P_{\Lambda, N}\left(A^{(2)}\right) & \leqslant \frac{\sum_{0<|k|<V^{-\eta}} E_{\Lambda, N}\left(n_{k}\right)}{\eta V} \\
& \leqslant \eta^{-1} V^{-1} \sum_{0<|k|<V^{-\eta}} \frac{1}{\mathrm{e}^{\varepsilon(k)+\nu}-1} .
\end{aligned}
$$

The right side vanishes as $V \rightarrow \infty$ : Indeed, this holds if the critical density is finite even if $\nu=0$; and we know that $\nu>0$ if the critical density is infinite.

Finally, A.10 implies that $P_{\Lambda, N}\left(n_{k} \geqslant i\right) \leqslant \mathrm{e}^{-\varepsilon(k) i}$. Since $\varepsilon(k)>a|k|^{2}$ for small $k$, we have for large $V$

$$
\begin{aligned}
P_{\Lambda, N}\left(A^{(3)}\right) & \leqslant \sum_{|k| \geqslant V^{-\eta}} P_{\Lambda, N}\left(n_{k} \geqslant V^{3 \eta}\right) \\
& \leqslant \sum_{|k| \geqslant V^{-\eta}} \mathrm{e}^{-\frac{1}{2} a V^{\eta}-\varepsilon(k)} \\
& \leqslant V \mathrm{e}^{-\frac{1}{2} a V^{\eta}} \sum_{k \in \Lambda^{*}} \frac{1}{V} \mathrm{e}^{-\varepsilon(k)} .
\end{aligned}
$$

The prefactor of the last line goes to 0 , while the sum converges to a finite Riemann integral. The whole expression vanishes in the limit.

\section{Appendix B. Convexity and Fourier positivity}

The case $d=1$ of the following lemma was used to provide an example of a onedimensional system with finite critical density. The result is certainly known - we were told that it may go back to Pólya - but it is easier to find a proof than a reference.

Lemma B.1. Let $g:(0, \infty) \rightarrow(0, \infty)$ such that $\int_{0}^{\infty} r^{d-1} g(r) \mathrm{d} r<\infty$, and such that $r^{d-1} g(r)$ is convex. Then the function $f(x)=g(|x|)$ on $\mathbb{R}^{d}$ has positive Fourier transform.

Proof. First, we show that for any convex function $h$ on $(0, \infty)$, we have

$$
\int_{n}^{n+1} h(u) \cos (2 \pi u) \mathrm{d} u \geqslant 0
$$

for any integer $n \geqslant 0$. It is enough to consider the case $n=0$; the general case follows from a change of variables (translates of convex functions are convex). We have

$$
\int_{0}^{1} h(u) \cos (2 \pi u) \mathrm{d} u=\int_{0}^{1 / 4}\left[h(u)-h\left(\frac{1}{2}-u\right)-h\left(\frac{1}{2}+u\right)+h(1-u)\right] \cos (2 \pi u) \mathrm{d} u .
$$

We used the fact that

$$
\cos (2 \pi u)=-\cos \left(2 \pi\left(\frac{1}{2}-u\right)\right)=-\cos \left(2 \pi\left(\frac{1}{2}+u\right)\right)=\cos (2 \pi(1-u)) .
$$

We now show that the bracket in $(\mathrm{B} .2)$ is positive. Let $\alpha=\frac{1 / 2}{1-2 u}$. Because $h$ is convex,

$$
\begin{aligned}
& h\left(\frac{1}{2}-u\right)=h(\alpha u+(1-\alpha)(1-u)) \leqslant \alpha h(u)+(1-\alpha) h(1-u), \\
& h\left(\frac{1}{2}+u\right)=h((1-\alpha) u+\alpha(1-u)) \leqslant(1-\alpha) h(u)+\alpha h(1-u) .
\end{aligned}
$$


This proves positivity of the bracket of $(\sqrt{\mathrm{B} .2})$, hence of $(\overline{\mathrm{B} .2})$ and $(\mathrm{B} .1)$. The case $d=1$ of Lemma B.1 follows, since $\widehat{f}(k)=2 \int_{0}^{\infty} f(r) \cos (2 \pi|k| r) \mathrm{d} r$.

For $d \geqslant 2$, let $\theta$ denote the angle between $x$ and $k$, and let $\Xi(\theta) \geqslant 0$ denote the measure of all remaining angles $(\Xi(\theta)=2$ for $d=2, \Xi(\theta)=2 \pi \sin \theta$ for $d=3)$. Then

$$
\begin{aligned}
\widehat{f}(k) & =\int_{0}^{\infty} r^{d-1} \mathrm{~d} r \int_{0}^{\pi} \Xi(\theta) \mathrm{d} \theta g(r) \cos (2 \pi|k| r \cos \theta) \\
& =2 \int_{0}^{\pi / 2} \frac{\Xi(\theta) \mathrm{d} \theta}{(|k| \cos \theta)^{d}} \int_{0}^{\infty} \mathrm{d} u u^{d-1} g\left(\frac{u}{|k| \cos \theta}\right) \cos (2 \pi u) .
\end{aligned}
$$

Now $u^{d-1} g\left(\frac{u}{|k| \cos \theta}\right)$ is convex in $u$ for given $|k|$ and given $0<\theta<\frac{\pi}{2}$ (scaled convex functions are convex). The latter integral is positive by (B.1).

\section{REFERENCES}

[1] D. Aldous, P. Diaconis, Longest increasing subsequences: from patience sorting to the Baik-DeiftJohansson theorem, Bull. Amer. Math. Soc. 36, 413-432 (1999)

[2] P. Arnold, G. Moore, BEC Transition Temperature of a Dilute Homogeneous Imperfect Bose Gas, Phys. Rev. Lett. 87, 120401 (2001)

[3] J. Baik, P. Deift, K. Johannson, On the distribution of the length of the longest increasing subsequence of random permutations J. Amer. Math. Soc. 12, 1119-1178 (1999)

[4] V. Betz, D. Ueltschi, in preparation

[5] G. Boland, J. V. Pulé, Long cycles in the infinite-range-hopping Bose-Hubbard model with hard cores, preprint (2008)

[6] E. Buffet, J. V. Pulé, Fluctuation properties of the imperfect Bose gas, J. Math. Phys. 24, 1608-1616 (1983)

[7] E. B. Davies, The thermodynamic limit for an imperfect boson gas, Commun. Math. Phys. 28, 69-86 (1972)

[8] P. Ferrari, M. Prähofer, H. Spohn, Stochastic growth in one dimension and Gaussian multi-matrix models, XIVth International Congress on Mathematical Physics, World Scientific 2005

[9] R. P. Feynman, Atomic theory of the $\lambda$ transition in Helium, Phys. Rev. 91, 1291-1301 (1953)

[10] K.-H. Fichtner, Random permutations of countable sets, Probab. Th. Rel. Fields 89, 35-60 (1991)

[11] D. Gandolfo, J. Ruiz, D. Ueltschi, On a model of random cycles, J. Stat. Phys. 129, 663-676 (2007)

[12] J. Ginibre, Some applications of functional integration in statistical mechanics, in "Mécanique statistique et théorie quantique des champs", Les Houches 1970, C. DeWitt and R. Stora eds, 327-427 (1971)

[13] C. Hainzl, R. Seiringer, General decomposition of radial functions on $\mathbb{R}^{n}$ and applications to $N$-body quantum systems, Lett. Math. Phys. 61, 75-84 (2002)

[14] V. A. Kashurnikov, N. V. Prokof'ev, B. V. Svistunov, Critical temperature shift in weakly interacting Bose gas, Phys. Rev. Lett. 87, 120402 (2001)

[15] B. Kastening, Bose-Einstein condensation temperature of a homogenous weakly interacting Bose gas in variational perturbation theory through seven loops, Phys. Rev. A 69, 043613 (2004)

[16] R. Kikuchi, $\lambda$ transition of liquid Helium, Phys. Rev. 96, 563-568 (1954)

[17] R. Kikuchi, H. H. Denman, C. L. Schreiber, Statistical mechanics of liquid He, Phys. Rev. 119, 18231831 (1960)

[18] J. L. Lebowitz, M. Lenci, H. Spohn, Large deviations for ideal quantum systems, J. Math. Phys. 41, 1224-1243 (2000)

[19] E. H. Lieb, R. Seiringer, J. P. Solovej, J. Yngvason, The mathematics of the Bose gas and its condensation, Oberwohlfach Seminars, Birkhäuser (2005); cond-mat/0610117

[20] K. Nho, D. P. Landau, Bose-Einstein condensation temperature of a homogeneous weakly interacting Bose gas: Path integral Monte Carlo study, Phys. Rev. A 70, 053614 (2004)

[21] A. Okounkov, Random matrices and random permutations, Internat. Math. Res. Notices 20, 1043-1095 (2000)

[22] A. Okounkov, The uses of random partitions, XIVth International Congress on Mathematical Physics, 379-403, World Scientific 2005 
[23] O. Penrose, L. Onsager, Bose-Einstein condensation and liquid Helium, Phys. Rev. 104, 576 (1956)

[24] E. L. Pollock, D. M. Ceperley, Path-integral computation of superfluid densities, Phys. Rev. B 36 , 8343-8352 (1987)

[25] A. Sütő, Percolation transition in the Bose gas, J. Phys. A 26, 4689-4710 (1993)

[26] A. Sütö, Percolation transition in the Bose gas II, J. Phys. A 35, 6995-7002 (2002)

[27] D. Ueltschi, Feynman cycles in the Bose gas, J. Math. Phys. 47, 123302 (2006)

[28] D. Ueltschi, Relation between Feynman cycles and off-diagonal long-range order, Phys. Rev. Lett. 97, 170601 (2006)

[29] D. Ueltschi, The model of interacting spatial permutations and its relation to the Bose gas, arXiv:0712.2443 (2007)

[30] V. A. Zagrebnov, J.-B. Bru, The Bogoliubov model of weakly imperfect Bose gas, Phys. Reports 350, 291-434 (2001)

Volker Betz and Daniel Ueltschi

Department of Mathematics

UNIVERSITY OF WARWICK

Coventry, CV4 7AL, England

http://www.maths.warwick.ac.uk/ betz/

http://www.ueltschi.org

E-mail address: v.m.betz@warwick.ac.uk, daniel@ueltschi.org 